Liviu I. Ignat · Enrique Zuazua

\title{
Convergence of a two-grid algorithm for the control of the wave equation
}

Received May 8, 2007 and in revised form June 22, 2007

\begin{abstract}
We analyze the problem of boundary observability of the finite-difference space semidiscretizations of the 2-d wave equation in the square. We prove the uniform (with respect to the meshsize) boundary observability for the solutions obtained by the two-grid preconditioner introduced by Glowinski [9]. Our method uses previously known uniform observability inequalities for low frequency solutions and a dyadic spectral time decomposition. As a consequence we prove the convergence of the two-grid algorithm for computing the boundary controls for the wave equation. The method can be applied in any space dimension, for more general domains and other discretization schemes.
\end{abstract}

\section{Introduction}

Let us consider the wave equation

$$
\begin{cases}y^{\prime \prime}-\Delta y=0 & \text { in } \Omega \times(0, T), \\ y=v \mathbf{1}_{\Gamma_{0}}(x) & \text { on } \Gamma \times(0, T), \\ y(0, x)=y^{0}(x), y^{\prime}(0, x)=y^{1}(x) & \text { in } \Omega,\end{cases}
$$

where $\Omega$ is the unit square $(0,1) \times(0,1) \subset \mathbb{R}^{2}$ and its boundary $\Gamma$ is decomposed as $\Gamma=\Gamma_{0} \cup \Gamma_{1}$ with

$$
\left\{\begin{array}{l}
\Gamma_{0}=\left\{\left(x_{1}, 1\right): x_{1} \in(0,1)\right\} \cup\left\{\left(1, x_{2}\right): x_{2} \in(0,1)\right\} \\
\Gamma_{1}=\left\{\left(x_{1}, 0\right): x_{1} \in(0,1)\right\} \cup\left\{\left(0, x_{2}\right): x_{2} \in(0,1)\right\} .
\end{array}\right.
$$

In equation (1.1), $y=y(t, x)$ is the state, ${ }^{\prime}$ is the time derivative and $v$ is a control function which acts on the system through the boundary $\Gamma_{0}$. Classical results of existence and uniqueness for solutions of nonhomogeneous evolution equations (see for instance [19]) show that for any $v \in L^{2}\left((0, T) \times \Gamma_{0}\right)$ and $\left(y^{0}, y^{1}\right) \in L^{2}(\Omega) \times H^{-1}(\Omega)$ equation (1.1) has a unique weak solution $\left(y, y^{\prime}\right) \in C\left([0, T], L^{2}(\Omega) \times H^{-1}(\Omega)\right)$.

Concerning the controllability of the above system the following exact controllability result is well known (see Lions [18]): Given $T>2 \sqrt{2}$ and $\left(y^{0}, y^{1}\right) \in L^{2}(\Omega) \times H^{-1}(\Omega)$

L. I. Ignat: Institute of Mathematics of the Romanian Academy, P.O. Box 1-764, RO-014700 Bucureşti, Romania; e-mail: liviu.ignat@gmail.com, web: http://www.imar.ro/ lignat E. Zuazua: Basque Center for Applied Mathematics, Bizkaia Technology Park, Building 208B, E-48170 Zamudio, Basque Country, Spain; e-mail: zuazua@bcamath.org, web: http://www.bcamath.org/zuazua/ 

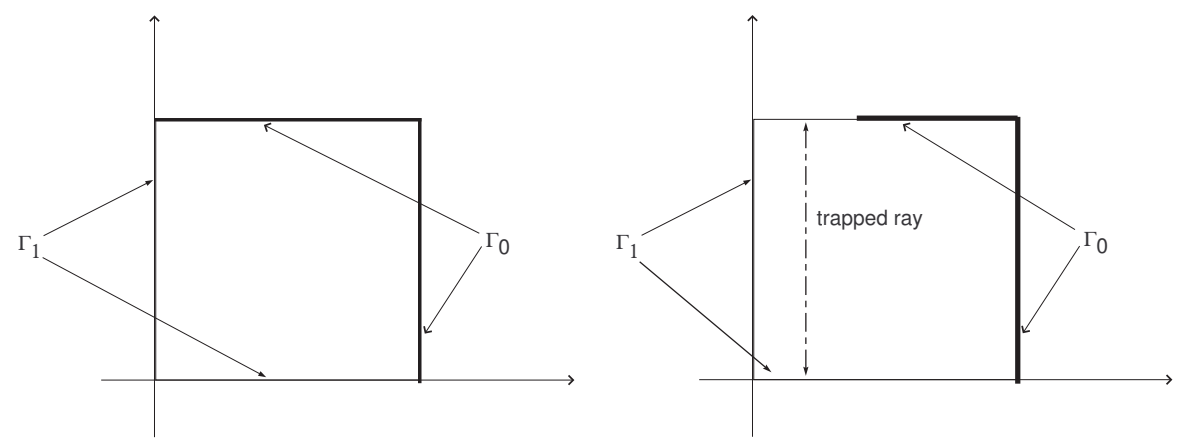

Fig. 1. Boundary of the domain $\Omega$ under consideration. $\Gamma_{0}$ is the subset where the control acts while $\Gamma_{1}$ is the one that remains uncontrolled.

there exists a control function $v \in L^{2}\left((0, T) \times \Gamma_{0}\right)$ such that the solution $y=y(t, x)$ of (1.1) satisfies

$$
y(T, \cdot)=y^{\prime}(T, \cdot)=0 .
$$

In fact, given $\left(y^{0}, y^{1}\right) \in L^{2}(\Omega) \times H^{-1}(\Omega)$ a control function $v$ of minimal $L^{2}((0, T) \times$ $\Gamma_{0}$ )-norm may be obtained by the so-called Hilbert Uniqueness Method (HUM, see [18]). It reduces the exact controllability problem to an equivalent observability property for the adjoint problem

$$
\begin{cases}u^{\prime \prime}-\Delta u=0 & \text { in } \Omega \times(0, T), \\ u=0 & \text { on } \Gamma \times(0, T), \\ u(0, x)=u^{0}(x), u_{t}(0, x)=u^{1}(x) & \text { in } \Omega .\end{cases}
$$

More precisely, the equivalent observability property is the following: For any $T>2 \sqrt{2}$ there exists $C(T)>0$ such that

$$
\left\|\left(u^{0}, u^{1}\right)\right\|_{H_{0}^{1}(\Omega) \times L^{2}(\Omega)}^{2} \leq C(T) \int_{0}^{T} \int_{\Gamma_{0}}\left|\frac{\partial u}{\partial n}\right|^{2} d \sigma d t
$$

for any $\left(u^{0}, u^{1}\right) \in H_{0}^{1}(\Omega) \times L^{2}(\Omega)$ where $u$ is the solution of $(1.3)$ with initial data $\left(u^{0}, u^{1}\right)$. Note that, rigorously speaking, the adjoint system should take the initial data at $t=T$. But, the wave equation being time reversible, this is irrelevant in what concerns the observability inequality (1.4).

The geometrical configuration considered in this paper is a particular instance in which the so-called Geometrical Control Condition (GCC) is satisfied. The GCC is a sharp condition guaranteeing the observability and controllability properties of the wave equation and it reads as follows: Every ray of geometrical optics that propagates in $\Omega$ and is reflected on its boundary $\partial \Omega$ intersects $\Gamma_{0}$ in time less than $T$. This condition has been proved to be sufficient and almost necessary in [2] (see also Burq [4] and Burq-Gérard [5]). In particular, the necessity of this condition is related to the fact that, around each 
ray that does not meet the observation/control region, one can always build concentrated gaussian beams making the observability inequality impossible.

In the particular case of the square domain we consider in this paper, the observability/controllability properties fail to hold if the control is supported on a set which is strictly smaller than two adjacent sides, as shown in Figure 1 (right). The control is located on a subset of two adjacent sides of the boundary, leaving a small horizontal subsegment uncontrolled. In that case GCC fails because of the existence of a ray trapped along a vertical segment bouncing back and forth on itself, without ever reaching the observation/control region.

In the case of the square domain the most natural configuration in which the GCC is satisfied is that in which the control is located on two full adjacent sides. In this case the needed observability inequalities can be obtained by the method of multipliers ([18]). This paper is devoted to analyzing this situation from a numerical analysis viewpoint.

The lower bound $2 \sqrt{2}$ on the observability time $T$ is due to the fact that, in this model, the velocity of propagation of waves is 1 and then, in order for (1.4) to be true, any perturbation of the initial data needs some time to reach the observation zone. The minimal time for this geometric configuration, $2 \sqrt{2}$, is twice the diameter of the domain, which is the largest travel time along the diagonal that a wave needs to get into the control region after bouncing on the opposite vertex.

The main objective of this paper is to prove the convergence of a numerical approximation algorithm for computing the control function $v$ of equation (1.1). This issue has been the object of intensive research in the past years. It is by now well known that the control of a stable numerical approximation scheme for (1.1) may diverge when the meshsize tends to zero. This is due to the unstabilizing effect of the high frequency numerical solutions [32]. Several techniques have been introduced as possible remedies to the instabilities produced by the high frequency spurious oscillations: Tikhonov regularization [9], filtering of the high frequencies [14], [31], [32], mixed finite elements [10], [7], [8] and the two-grid algorithm [25], [20].

Possibly the one which is most systematic and convenient for practical implementations is the two-grid algorithm proposed by Glowinski [9]. The method consists in relaxing the controllability requirement on numerical solutions by considering only its projection over a coarser grid. In what concerns the observability inequality (1.4) the method consists in analyzing the discrete or semidiscrete version of (1.4) for the solutions of the numerical approximation scheme, but only for initial data obtained through a two-grid preconditioning. To be more precise, the two-grid method consists in using a coarse and a fine grid, and interpolating the initial data for the numerical approximation of (1.3) from the coarse $G_{c}$ grid to the fine one $G_{f}$. This method attenuates the short wave-length components of the initial data, which are responsible for the spurious high frequency oscillations.

The main goal of the paper is to rigorously prove the convergence of this algorithm in the context of the semidiscrete finite-difference approximation scheme for the wave equation in the square. The key ingredient of the proof is an inequality similar to (1.4) at the semidiscrete level, independent of the mesh-size, for the two-grid data mentioned above. 
Throughout the paper we deal with the two-dimensional case but all the arguments we present here work in any space dimension and can also be applied to other numerical schemes, both semidiscrete and fully discrete.

Our main contribution is to develop a dyadic decomposition argument that allows reducing the problem to considering classes of solutions in which the high frequency components have been filtered, a situation that was already dealt with in the literature.

To fix ideas, consider the finite-difference semidiscretization of (1.3). Given $N \in \mathbb{N}$ we set $h=1 /(N+1), \Omega_{h}=\Omega \cap h \mathbb{Z}^{2}$ and $\Gamma_{h}=\Gamma \cap h \mathbb{Z}^{2}$. In the same manner we define $\Gamma_{0 h}$ and $\Gamma_{1 h}$. The finite-difference semidiscretization of system 1.1 is as follows:

$$
\begin{cases}y_{h}^{\prime \prime}-\Delta_{h} y_{h}=0 & \text { in } \Omega_{h} \times(0, T) \\ y_{h}=v_{h} \mathbf{1}_{\Gamma_{0 h}} & \text { on } \Gamma_{h} \times(0, T) \\ y_{h}(0)=y_{h}^{0}, \quad y_{h}^{\prime}(0)=y_{h}^{1} & \text { in } \Omega_{h}\end{cases}
$$

where the initial data $\left(y_{h}^{0}, y_{h}^{1}\right)$ are approximations of $\left(y^{0}, y^{1}\right)$ and $\Delta_{h}$ is the five-point approximation of the laplacian:

$$
\left(\Delta_{h} u\right)_{j, k}=\frac{u_{j-1, k}-2 u_{j, k}+u_{j+1, k}}{h^{2}}+\frac{u_{j, k-1}-2 u_{j, k}+u_{j, k+1}}{h^{2}} .
$$

For the homogeneous wave equation 1.3 we consider the following numerical scheme:

$$
\begin{cases}u_{h}^{\prime \prime}-\Delta_{h} u_{h}=0 & \text { in } \Omega_{h} \times[0, T] \\ u_{h}=0, & \text { on } \Gamma_{h} \times(0, T) \\ u_{h}(0)=u_{h}^{0}, u_{h}^{\prime}(0)=u_{h}^{1} & \text { in } \Omega_{h}\end{cases}
$$

To simplify the presentation, whenever it is not strictly necessary, we will avoid the subscript $h$ in the notation of the solution $u_{h}$.

Let us now introduce the discrete energy associated with system (1.6):

$$
\mathcal{E}_{h}(t)=\frac{h^{2}}{2} \sum_{j, k=0}^{N}\left[\left|u_{j, k}^{\prime}(t)\right|^{2}+\left|\frac{u_{j+1, k}(t)-u_{j, k}(t)}{h}\right|^{2}+\left|\frac{u_{j, k+1}(t)-u_{j, k}(t)}{h}\right|^{2}\right] .
$$

It is easy to see that the energy remains constant in time, i.e.

$$
\mathcal{E}_{h}(t)=\mathcal{E}_{h}(0), \quad \forall 0<t<T,
$$

for every solution of 1.6 . by

Following [1], the discrete version of the energy observed on the boundary $\Gamma_{0}$ is given

$$
\int_{0}^{T} \int_{\Gamma_{0}}\left|\frac{\partial u}{\partial n}\right|^{2} d \sigma d t \simeq \int_{0}^{T}\left[h \sum_{j=1}^{N}\left|\frac{u_{j, N}}{h}\right|^{2}+h \sum_{k=1}^{N}\left|\frac{u_{N, k}}{h}\right|^{2}\right] d t
$$

For any $j, k=1, \ldots, N$, we define

$$
\begin{aligned}
\left(\partial_{n}^{h} u\right)_{j, N+1} & :=-\frac{u_{j, N}}{h}, & \left(\partial_{n}^{h} u\right)_{N+1, k} & :=-\frac{u_{N, k}}{h}, \\
\left(\partial_{n}^{h} u\right)_{j, 0} & :=-\frac{u_{j, 1}}{h}, & \left(\partial_{n}^{h} u\right)_{0, k}: & =-\frac{u_{1, k}}{h} .
\end{aligned}
$$


Also, in order to simplify the presentation, we shall use integrals to denote discrete sums, i.e.

$$
\int_{\Omega_{h}} u d \Omega_{h}:=h^{2} \sum_{\mathbf{j} h \in \Omega_{h}} u_{\mathbf{j}}, \quad \int_{\Gamma_{h}} u d \Gamma_{h}:=h \sum_{\mathbf{j} h \in \Gamma_{h}} u_{\mathbf{j}} .
$$

and

$$
\int_{\Gamma_{0 h}}\left|\partial_{n}^{h} u\right|^{2} d \Gamma_{0 h}:=h \sum_{j=1}^{N}\left|\frac{u_{j, N}}{h}\right|^{2}+h \sum_{k=1}^{N}\left|\frac{u_{N, k}}{h}\right|^{2} .
$$

The discrete version of (1.4) is then an inequality of the form

$$
\mathcal{E}_{h}(0) \leq C_{h}(T) \int_{0}^{T} \int_{\Gamma_{0 h}}\left|\partial_{n}^{h} u\right|^{2} d \Gamma_{0 h} d t .
$$

System (1.6) being finite-dimensional, it is easy to see that the so-called Kalman rank condition is satisfied, and consequently, for all $T, h>0$ there exists a constant $C_{h}(T)$ such that inequality (1.11) holds for all solutions of equation (1.3). But, as proved in [31], for all $T>0$ the best constant $C_{h}(T)$ necessarily blows up as $h \rightarrow 0$. The blow-up of the observability constant is due to two main reasons. First, the discrete version of the normal derivative in 1.9 ) is too weak to capture the energy of the high frequency monochromatic waves. This could be compensated by making more accurate boundary measurements, but this would not suffice due to the fact that the numerical scheme develops high frequency wave packets whose group velocity is of the order of $h$. These high frequency solutions are such that the energy concentrated on the boundary $\Gamma_{0 h}$ is asymptotically smaller than the total energy. This phenomenon was already observed by R. Glowinski et al. [9], [11], [12]. Using a wave-packet construction it can be shown that the observability constant $C_{h}(T)$ blows up exponentially as $h \rightarrow 0$. We refer to Micu [22] for a detailed proof in the $1-\mathrm{d}$ case based on explicit estimates of families biorthogonal to the complex exponentials entering the Fourier development of solutions.

As proved in [31], inequality (1.11) holds uniformly in a class of low frequency solutions (initial data where the spurious high frequency modes have been filtered) provided the time $T$ is large enough depending on the frequencies under consideration. In Section 2 we will make this concept precise and recall this result. The main result of this paper, stated in Section 2, guarantees that, once (1.11) holds uniformly for a class of low frequency solutions, it also holds for all solutions in an extended class of initial data whose energy is controlled by their projection on the previous low frequency components. As we shall see, the class of initial data for (1.6) obtained through the two-grid approach fulfills these requirements. Accordingly, we shall deduce that for $T>0$ large enough inequality (1.11) holds uniformly (i.e. with a constant $C_{h}(T)$ which is independent of $h$ ) in this class of two-grid data. As a consequence, we will conclude that system (1.5) is uniformly controllable in the sense that the projections of the states onto the coarse grid are controllable with controls that remain bounded as $h \rightarrow 0$. Furthermore, these controls converge to those of 11.1 as the mesh-size $h$ goes to 0 .

In the one-dimensional case, the two-grid method was analyzed by Negreanu and Zuazua [25] with a discrete multiplier approach. The authors considered two meshes with 
quotient $1 / 2$ and proved the convergence of the method as $h \rightarrow 0$ for $T>4$. The same two-grid method has been considered in a more recent work by Loreti and Mehrenberger [20], where the authors use a fine extension of Ingham's inequality to obtain a sharp time of uniform observability, namely $T>2 \sqrt{2}$. However, as far as we know, there is no proof of the uniform observability in the two-dimensional case. The main goal of this paper is to give the first complete proof of convergence of the method in a multidimensional setting.

In contrast with the strategy adopted in [25] we choose two grids with the quotient of their sizes being $1 / 3$. This is done for merely technical reasons, which we shall describe in the last section, and one may expect the same result to hold when the ratio of the grids is $1 / 2$. The problem is open in the multidimensional case for the mesh-ratio $1 / 2$.

Our method, which consists in using the already well known observability inequality for a class of low frequency data and a dyadic time spectral decomposition of the solutions, works in any space dimension and for other discretization schemes. We shall return to this issue in Section 8 where we shall briefly discuss the possible extensions of the results of this paper.

The two-grid method has also been used in other contexts to filter the unwanted effect of high frequency numerical solutions. For instance, in [13], it was employed with two meshes of mesh-ratio $1 / 4$ when proving dispersive estimates for conservative semidiscrete approximation schemes of the Schrödinger equation. There, using the mesh-ratio $1 / 4$ was necessary. Here, as mentioned above, the result might well hold for $1 / 2$ as in $1-d$ but, for technical reasons, we prove it only for $1 / 3$. Our techniques also allow showing the convergence of the method for meshes with mutual ratio $1 / p$ for any $p \geq 3$. We present the case $1 / 3$ since it is the one in which the amount of filtering is minimal.

Indeed, when diminishing the ratio between grids, the attenuation that the two-grid algorithm introduces on the high frequency component of the solutions is enhanced and the energy is concentrated on lower frequencies for which the velocity of propagation becomes closer to that of the continuous wave equation. It is therefore natural to expect that proving the uniform observability will be easier for smaller grid ratios. When doing that one may also expect that the time of control will get closer to the optimal one of the continuous wave equation. Both facts will be explicitly established through our analysis.

The rest of the paper is organized as follows. In Section 2 we introduce the spaces $K_{h}^{M}(\gamma)$ consisting of all the discrete functions $(\varphi, \psi)$ such that their norm is controlled by the one of their projection on a suitable low frequency component, and state the core result of this paper: the uniform observability inequality for data that belong to these spaces. In Section 3 we will introduce the space $V^{h}$ of functions defined on the fine grid $G^{h}$ as linear interpolation of functions defined on the coarse one $G^{3 h}$. We prove that 1.11) holds uniformly for all $T>4 \sqrt{2}$, in the class of two-grid initial data $V^{h} \times V^{h}$. Section 4 is devoted to the proof of the main result of this paper, namely Theorem 2.1 . using the dyadic decomposition argument. The last sections are devoted to proving the convergence of controls. More precisely, in Section 5 we construct semidiscrete control functions $v_{h}$ for (1.5) that approximate the control function $v$ in 1.1 . Section 6 contains convergence results for the uncontrolled problem that will be used in Section 7 to prove the convergence in $L^{2}\left((0, T) \times \Gamma_{0}\right)$ of the functions $v_{h}$, constructed before, towards the continuous one $v$. In the last section we comment on the main result of the paper, on how 
it can be used or improved, and on its limitations, and we also formulate a number of open problems. The paper also has two appendices containing some technical lemmas and the Fourier analysis of the discrete functions obtained by a two-grid algorithm.

\section{The observability problem}

To make our statements precise, let us consider the eigenvalue problem associated to (1.6):

$$
\begin{cases}-\Delta_{h} \varphi_{h}=\lambda \varphi_{h} & \text { in } \Omega_{h} \\ \varphi_{h}=0 & \text { on } \Gamma_{h}\end{cases}
$$

Define $\Lambda_{N}:=[1, N]^{2} \cap \mathbb{Z}^{2}$. The eigenvalues and eigenvectors of system 2.1] are

$$
\lambda_{\mathbf{j}}(h)=\frac{4}{h^{2}}\left[\sin ^{2}\left(\frac{j_{1} \pi h}{2}\right)+\sin ^{2}\left(\frac{j_{2} \pi h}{2}\right)\right], \quad \mathbf{j}=\left(j_{1}, j_{2}\right) \in \Lambda_{N},
$$

and

$\varphi_{h}^{\mathbf{j}}(\mathbf{k})=2 \sin \left(j_{1} k_{1} \pi h\right) \sin \left(j_{2} k_{2} \pi h\right), \mathbf{k}=\left(k_{1}, k_{2}\right) \in[0, N+1]^{2} \cap \mathbb{Z}^{2}, \mathbf{j}=\left(j_{1}, j_{2}\right) \in \Lambda_{N}$.

The vectors $\left\{\varphi_{h}^{\mathbf{j}}\right\}_{\mathbf{j} \in \Lambda_{N}}$ form a basis for the discrete functions $\phi_{h}$ defined on $G^{h}=\Omega_{h} \cup \Gamma_{h}$ and vanishing on its boundary, allowing us to write, for any discrete function $\phi_{h}$,

$$
\phi_{h}=\sum_{\mathbf{j} \in \Lambda_{N}} \widehat{\phi}_{h}(\mathbf{j}) \varphi_{h}^{\mathbf{j}},
$$

where $\widehat{\phi}(\mathbf{j})=\left(\phi_{h}, \varphi_{h}^{\mathbf{j}}\right)_{h},(\cdot, \cdot)_{h}$ being the inner product in $l^{2}\left(\Omega_{h}\right)$ :

$$
(u, v)_{h}=h^{2} \sum_{\mathbf{k} h \in \Omega_{h}} u(\mathbf{k}) v(\mathbf{k}) .
$$

In view of this representation, for every $s \in \mathbb{R}$, we will denote by $\mathcal{H}_{h}^{s}\left(\Omega_{h}\right)$ the space of all functions defined on the grid $G^{h}$, endowed with the norm

$$
\left\|\phi_{h}\right\|_{s, h}=\left(\sum_{\mathbf{j} \in \Lambda_{N}} \lambda_{\mathbf{j}}^{2 s}(h)\left|\widehat{\phi}_{h}(\mathbf{j})\right|^{2}\right)^{1 / 2} .
$$

Let $\left\{\widehat{u}_{h}^{0}(\mathbf{j})\right\}_{\mathbf{j} \in \Lambda_{N}}$ and $\left\{\widehat{u}_{h}^{1}(\mathbf{j})\right\}_{\mathbf{j} \in \Lambda_{N}}$ be the coefficients of the initial data $\left(u_{h}^{0}, u_{h}^{1}\right)$ of system 1.6 in the basis $\left\{\varphi_{h}^{\mathbf{j}}\right\}_{\mathbf{j} \in \Lambda_{N}}$. Then the solution $u_{h}$ is given by

$$
u_{h}(t)=\frac{1}{2} \sum_{\mathbf{j} \in \Lambda_{N}}\left[e^{i t \omega_{\mathbf{j}}(h)} \widehat{u}_{\mathbf{j}+}^{h}+e^{-i t \omega_{\mathbf{j}}(h)} \widehat{u}_{\mathbf{j}-}^{h}\right] \varphi_{h}^{\mathbf{j}},
$$

where $\omega_{\mathbf{j}}(h)=\sqrt{\lambda_{\mathbf{j}}(h)}$ and

$$
\widehat{u}_{\mathbf{j} \pm}^{h}=\widehat{u}_{h}^{0}(\mathbf{j}) \pm \frac{\widehat{u}_{h}^{1}(\mathbf{j})}{i \sqrt{\lambda_{\mathbf{j}}(h)}}
$$




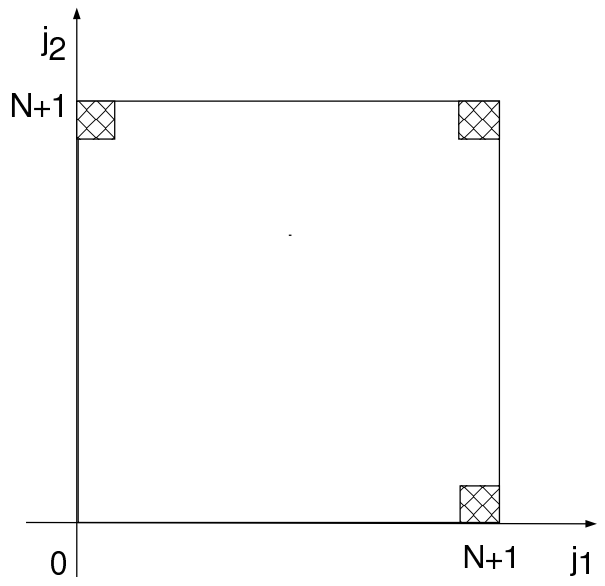

Fig. 2. The three dashed corners contain solutions whose group velocity is of order $h$.

Using the above notations, the energy of the system introduced in (1.7) is conserved in time and satisfies

$$
\mathcal{E}_{h}\left(u_{h}\right)=\sum_{\mathbf{j} \in \Lambda_{N}} \omega_{\mathbf{j}}^{2}(h)\left(\left|\widehat{u}_{\mathbf{j}+}^{h}\right|^{2}+\left|\widehat{u}_{\mathbf{j}-}^{h}\right|^{2}\right) .
$$

Let us introduce the class of filtered solutions of 1.6 in which the high frequencies have been truncated or filtered. More precisely, for any $0<\gamma \leq 2 \sqrt{2}$ we set

$$
I_{h}(\gamma)=\left\{u_{h}: u_{h}=\sum_{\omega_{\mathbf{j}}(h) \leq \gamma / h} \widehat{u}_{\mathbf{j}}^{h} \varphi_{h}^{\mathbf{j}} \text { with } \widehat{u}_{\mathbf{j}}^{h} \in \mathbb{C}\right\} .
$$

The class $I_{h}(\gamma)$ has been intensively used for control problems ([15], [3], [16]) and the dispersive properties of PDE's ([6]). For any solution $u_{h}$ of equation (1.6) we denote by $\Pi_{h}^{\gamma} u_{h}$ its projection on the space $I_{h}(\gamma)$, which consists simply in restricting the Fourier expansion (2.3) to the class of indices in $I_{h}(\gamma)$ for which $\omega_{\mathbf{j}}(h) \leq \gamma / h$.

The uniform observability in the class $I_{h}(\gamma)$ has been analyzed in [31] by the multiplier technique. In that article it is shown that for any $0<\gamma<2$ and

$$
T>T(\gamma)=\frac{8 \sqrt{2}}{4-\gamma^{2}}
$$

there exists $C(\gamma, T)>0$ such that

$$
\mathcal{E}_{h}\left(u_{h}\right) \leq C(\gamma, T) \int_{0}^{T} \int_{\Gamma_{0 h}}\left|\partial_{n}^{h} u_{h}(t)\right|^{2} d \Gamma_{0 h} d t
$$

holds for every solution $u$ of 1.6 in the class $I_{h}(\gamma)$ and $h>0$. This observability result will be systematically used along the paper. The choice of the filtering parameter $\gamma<2$ 


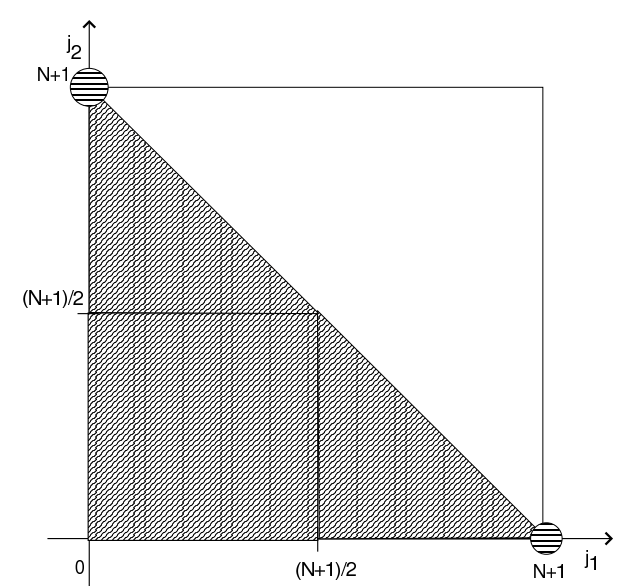

Fig. 3. The dashed area below the diagonal of the square represents the frequencies involved in $I_{h}(2)$. The two circles in the corners correspond to frequencies with group velocity of order $h$ that enter the class $I_{h}(2)$ but are excluded for filtering parameter $\gamma<2$.

in [31] is sharp. More precisely, for $\gamma=2$ and any $T>0$ it was shown that there is no constant $C(T)$ (see [31]) such that (2.6) holds for all solutions $u_{h}$ of (1.6), uniformly in $h$ :

$$
\sup _{u_{h} \in I_{h}(2)} \frac{\mathcal{E}_{h}\left(u_{h}\right)}{\int_{0}^{T} \int_{\Gamma_{0 h}}\left|\partial_{n}^{h} u_{h}(t)\right|^{2} d \Gamma_{0 h} d t} \rightarrow \infty, \quad h \rightarrow 0 .
$$

This is a consequence of the presence of solutions which have group velocity of order $h$ and spend a time of order $1 / h$ to reach the boundary. In Figure 2 we can see the areas of the spectrum in which these solutions with group velocity of order $h$ can occur and in Figure 3 we illustrate how some of them enter the class of filtered solutions $I_{h}(\gamma)$ for $\gamma=2$. The classes $I_{h}(\gamma)$ make sense for all $0<\gamma \leq 2 \sqrt{2}$ in view of the obvious spectral bound $\lambda_{\mathbf{j}}(h) \leq 8 / h^{2}$, which immediately holds as a consequence of the explicit expression (2.2). But, obviously, the observability estimate 2.6. fails to be uniform in $I_{h}(\gamma)$ for all $2 \leq \gamma \leq 2 \sqrt{2}$, because it actually fails for $\gamma=2$.

The main goal of this paper is to extend this uniform observability inequality to a more general class of initial data obtained through the two-grid filtering strategy. In this class the high frequency components do not vanish but a careful analysis shows that their energy is dominated by the low frequency ones.

To be more precise, let $\Pi_{h}^{\gamma}$ be the orthogonal projection of discrete functions over the subspace $I_{h}(\gamma)$. Let us now fix $M>0$. For any $0<\gamma \leq 2 \sqrt{2}$ we define $K_{h}^{M}(\gamma)$ as the subspace of $\mathcal{H}_{h}^{1}\left(\Omega_{h}\right) \times \mathcal{H}_{h}^{0}\left(\Omega_{h}\right)$ consisting of all the discrete functions $(\varphi, \psi)$ such that their square norm is controlled by the one of their projection on $I_{h}(\gamma)$ by a factor of $M$ :

$$
K_{h}^{M}(\gamma)=\left\{(\varphi, \psi):\|\varphi\|_{1, h}^{2}+\|\psi\|_{0, h}^{2} \leq M\left(\left\|\Pi_{h}^{\gamma} \varphi\right\|_{1, h}^{2}+\left\|\Pi_{h}^{\gamma} \psi\right\|_{0, h}^{2}\right)\right\} .
$$


We point out that the conservation of energy 1.8 guarantees that the solutions of equation (1.6) with initial data $\left(u_{h}^{0}, u_{h}^{1}\right) \in K_{h}^{M}(\gamma)$ satisfy

$$
\mathcal{E}_{h}\left(u_{h}\right) \leq M \mathcal{E}_{h}\left(\Pi_{h}^{\gamma} u_{h}\right)
$$

Therefore $K_{h}^{M}(\gamma)$ is stable under the flow and $\left(u_{h}(t), u_{h}^{\prime}(t)\right) \in K_{h}^{M}(\gamma)$ for any $t \geq 0$.

The main result of this section is given by the following theorem.

Theorem 2.1. Let $\gamma>0$ and $M>0$ be given. Assume the existence of a time $T(\gamma)$ such that for all $T>T(\gamma)$ there exists a positive constant $C=C(\gamma, T)$, independent of $h$, such that

$$
\mathcal{E}_{h}\left(u_{h}\right) \leq C \int_{0}^{T} \int_{\Gamma_{0 h}}\left|\partial_{n}^{h} u_{h}(t)\right|^{2} d \Gamma d t
$$

for all $\left(u_{h}^{0}, u_{h}^{1}\right) \in I_{h}(\gamma)$. Then for all $T>T(\gamma)$ there exists a positive constant $C=$ $C_{1}(\gamma, T, M)$, such that 2.9. holds for all solutions $u_{h}$ of problem 1.6 with initial data $\left(u_{h}^{0}, u_{h}^{1}\right) \in K_{h}^{M}(\gamma)$ and $h>0$.

Remark 2.1. According to Theorem 2.1 the uniform observability inequality can be automatically transferred from $I_{h}(\gamma)$ to $K_{h}^{M}(\gamma)$. Let us briefly explain the main difficulty of the proof of Theorem 2.1. Inequalities 2.8) and 2.9) show that the uniform boundary observability inequality

$$
\mathcal{E}_{h}\left(u_{h}\right) \leq C(T) \int_{0}^{T} \int_{\Gamma_{0 h}}\left|\partial_{n}^{h} \Pi_{h}^{\gamma} u_{h}\right|^{2} d \Gamma_{0 h} d t
$$

holds in the class $K_{h}^{M}(\gamma)$ as well. But, unfortunately, the right side term cannot be estimated directly in terms of the energy of the solution $u_{h}$ measured at the boundary $\Gamma_{0 h}$ :

$$
\int_{0}^{T} \int_{\Gamma_{0 h}}\left|\partial_{n}^{h} u_{h}\right|^{2} d \Gamma_{0 h} d t
$$

A careful analysis is required to show that estimate. The essential contribution of this article is to show how this may be done by means of a dyadic decomposition.

Remark 2.2. In the proof of the above theorem we use the so-called "direct inequality". In fact, it is well known that (see [31]) for any $T>0$ there exists a constant $C(T)$, independent of $h$, such that

$$
\int_{0}^{T} \int_{\Gamma_{0 h}}\left|\partial_{n}^{h} u_{h}\right|^{2} d \Gamma_{0 h} d t \leq C(T) \mathcal{E}_{h}\left(u_{h}\right)
$$

for all solutions $u$ of the semidiscrete system 1.6 and for all $h>0$.

Remark 2.3. The same result holds if the two-grid filtered initial data are taken at any time $t_{0} \in[0, T]$. In this sense our method of proof is more robust than the one in [20] which makes a distinction between observability in the interval $[0, T]$ or $[-T / 2, T / 2]$ that our arguments show is not necessary.

Since the proof of Theorem 2.1 is quite laborious it will be postponed until Section 4 


\section{A two-grid method}

In this section we describe a two-grid method that naturally produces classes of initial data of the form $K_{h}^{M}(\gamma)$. In view of Theorem 2.1 this will allow us to show immediately uniform observability estimates for these classes of two-grid data.

The two-grid algorithm we propose is the following: Let $N$ be such that $N \equiv 2$ $(\bmod 3)$ and $h=1 /(N+1)$. We introduce a coarse grid of mesh-size $3 h$ :

$$
G^{3 h}: \quad x_{\mathbf{j}}=3 \mathbf{j} h, \quad \mathbf{j} \in[0,(N+1) / 3]^{2} \cap \mathbb{Z}^{2},
$$

and a fine one of size $h$ :

$$
G^{h}: \quad y_{\mathbf{j}}=\mathbf{j} h, \quad \mathbf{j} \in[0, N+1]^{2} \cap \mathbb{Z}^{2} .
$$

We consider the space $V^{h}$ of all functions $\varphi$ defined on the fine grid $G^{h}$ as a linear interpolation of the functions $\psi$ defined on the coarse grid $G^{3 h}$. To be more precise, let us consider the spaces $\mathcal{G}_{h}$ and $\mathcal{G}_{3 h}$ of all the functions defined on the fine and coarse grids $G^{h}$ and $G^{3 h}$ respectively. We also introduce the extension operator $\Pi_{h}^{3 h}$ which associates to any function $\psi \in \mathcal{G}_{3 h}$ a new function $\Pi_{h}^{3 h} \psi \in \mathcal{G}_{h}$ obtained by an interpolation process:

$$
\left(\boldsymbol{\Pi}_{h}^{3 h} \psi\right)_{\mathbf{j}}=\left(\mathbf{P}_{3 h}^{1} \psi\right)(\mathbf{j} h), \quad \mathbf{j} \in \mathbb{Z}^{2},
$$

where $\mathbf{P}_{3 h}^{1} \psi$ is the piecewise multi-linear interpolator of $\psi \in \mathcal{G}_{3 h}$. We then define $V^{h}=$ $\boldsymbol{\Pi}_{h}^{3 h}\left(\mathcal{G}_{3 h}\right)$, the image of the operator $\boldsymbol{\Pi}_{h}^{3 h}$. Obviously this constitutes a subspace of slowly oscillating discrete functions defined on the fine grid $G^{h}$. Examples of this interpolation process are given in Figure 4.

We now define another class of filtered functions, better adapted to the spectral analysis of the two-grid ones. For any $\mathbf{j}=\left(j_{1}, j_{2}\right) \in \mathbb{Z}^{2}$, we denote its maximal component by $\|\mathbf{j}\|_{\infty}=\max \left\{j_{1}, j_{2}\right\}$. For any $0<\eta \leq 1$ we set

$$
J_{h}(\eta)=\left\{u_{h}: u_{h}=\sum_{\|\mathbf{j}\|_{\infty} \leq \eta(N+1)} \widehat{u}_{\mathbf{j}}^{h} \varphi_{h}^{\mathbf{j}} \text { with } \widehat{u}_{\mathbf{j}}^{h} \in \mathbb{C}\right\},
$$

and for any solution $u_{h}$ of 1.6$)$ we denote by $\Upsilon_{h}^{\eta} u_{h}$ its projection on the space $J_{h}(\eta)$.

The class of filtered solutions $I_{\gamma}(h)$, introduced in Section 2, is obtained through a filtering process along the level curves of $\omega_{\mathbf{j}}(h)$. The second one, leading to the space $J_{h}(\eta)$, consists in filtering the range of indices $\mathbf{j}$ to a square with length side $\eta(N+1)$. Observe that in dimension one there exists a one-to-one correspondence between the two classes. In dimension two, excepting the case $\gamma=2 \sqrt{2}$, which corresponds to $\eta=1$, there is no one-to-one correspondence. However, the two classes can be easily compared with each other by analyzing the shape of the level curves of $\omega_{\mathbf{j}}(h)$. In Figure 5 we can see the support of the discrete functions in the frequency domain for the classes $J_{h}(1 / 3)$ and $I_{h}(\sqrt{2})$ that occur in the analysis of our two-grid method.

The second class of filtered data $J_{h}(\eta)$ is better adapted to analyze the two-grid discrete functions. In fact, we will prove that the total energy of a solution $u_{h}$ of (1.6) with 

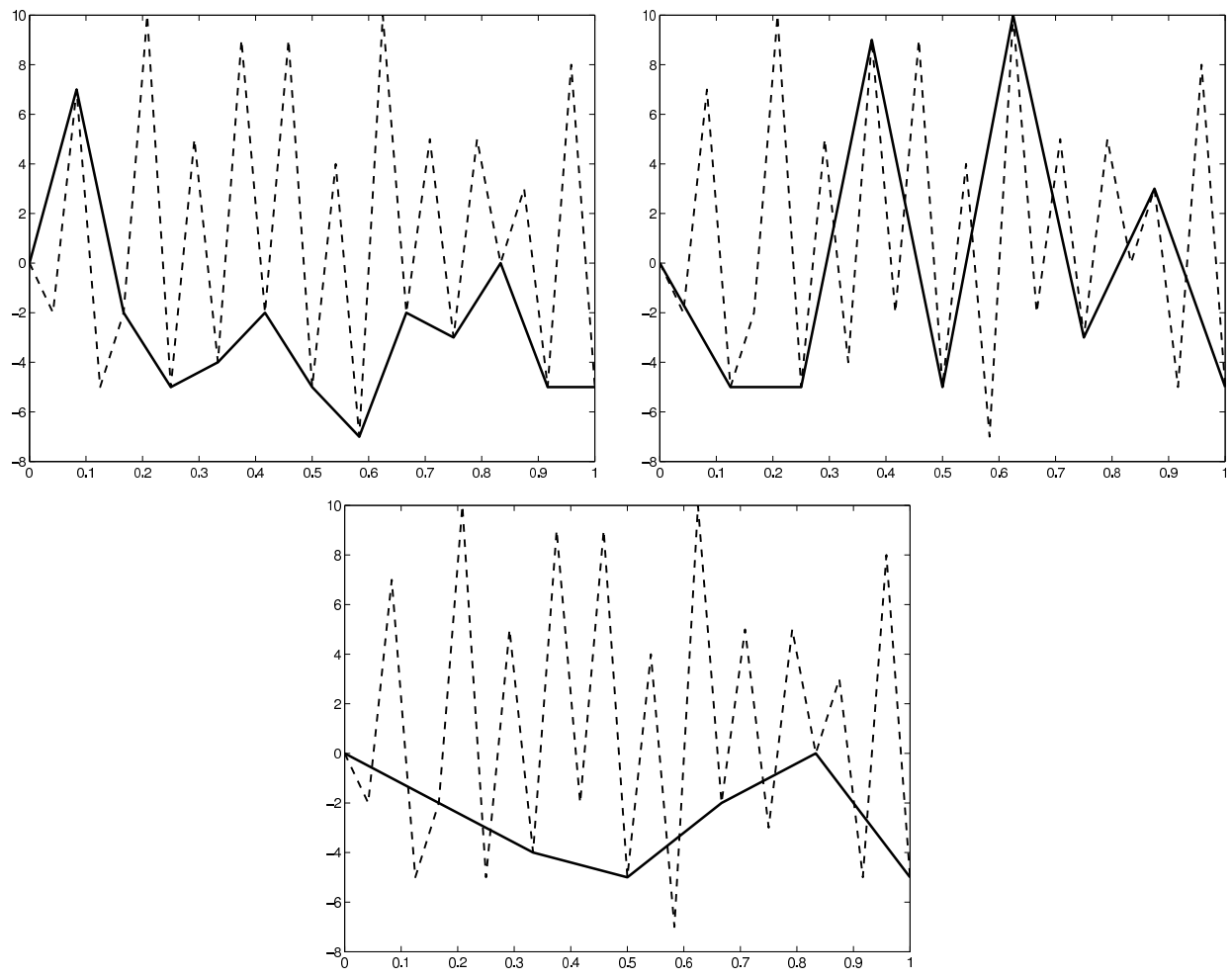

Fig. 4. The dashed line is the original discrete function $u$. From left to write the new functions $\Pi_{h}^{2 h} u, \Pi_{h}^{3 h} u, \Pi_{h}^{4 h} u$ respectively.
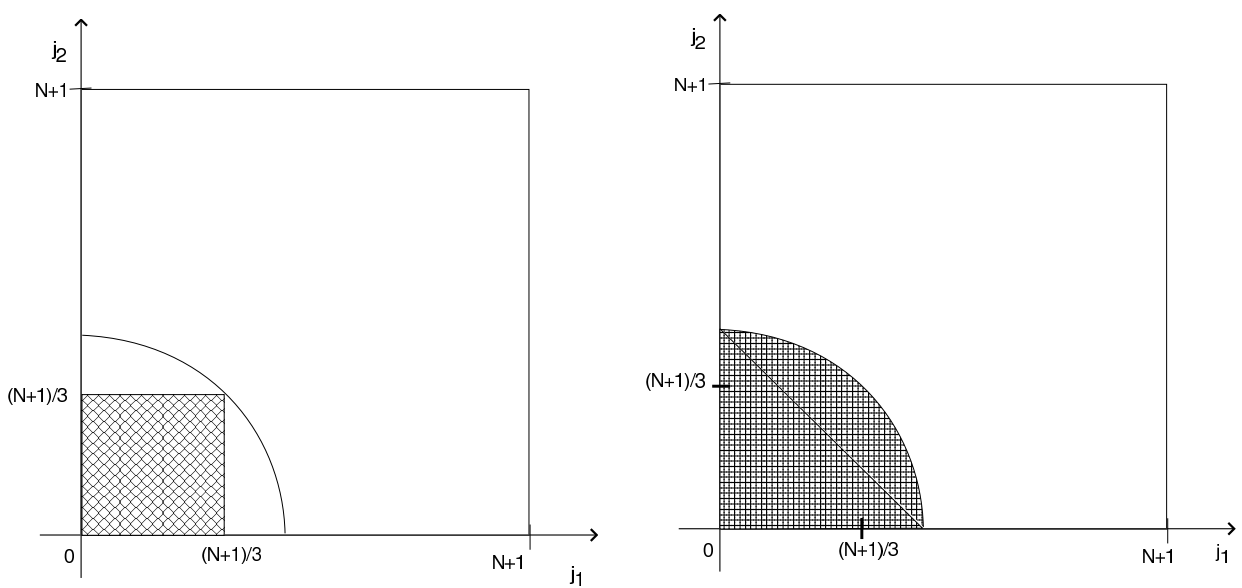

Fig. 5. Left: the dashed area represents the frequencies $\omega_{\mathbf{j}}(h), \mathbf{j} \in \Lambda_{(N+1) / 3}$; right: the dashed area represents the frequencies involved in $I_{h}(\sqrt{2})$. 
initial data in the space $V^{h} \times V^{h}$ is bounded above by the energy of its projection on the space $J_{h}(1 / 3)$ :

$$
\mathcal{E}_{h}\left(u_{h}\right) \leq M \mathcal{E}_{h}\left(\Upsilon_{h}^{1 / 3} u_{h}\right)
$$

for some positive constant $M$, independent of $h$. We point out that it is sufficient to prove this bound for $t=0$, i.e. for the initial data, and use the fact that the space $J_{h}(1 / 3)$ remains invariant under the semidiscrete flow to deduce that $(3.2)$ holds for all $t>0$. More precisely, it is sufficient to show that, for $\left(u_{h}^{0}, u_{h}^{1}\right) \in V^{h} \times V^{h}$,

$$
\left\|u_{h}^{0}\right\|_{1, h}^{2} \leq M\left\|\Upsilon_{h}^{1 / 3} u_{h}^{0}\right\|_{1, h}^{2}
$$

and

$$
\left\|u_{h}^{1}\right\|_{0, h}^{2} \leq M\left\|\Upsilon_{h}^{1 / 3} u_{h}^{1}\right\|_{0, h}^{2} .
$$

Observe that any $\omega_{\mathbf{j}}(h)$ with $\|\mathbf{j}\|_{\infty} \leq(N+1) / 3$ satisfies

$$
\omega_{\mathbf{j}}(h) \leq\left(\frac{8}{h^{2}} \sin ^{2}\left(\frac{\pi}{6}\right)\right)^{1 / 2} \leq \frac{\sqrt{2}}{h},
$$

and thus, in view of (3.2), the energy of $u_{h}$ is bounded above by the energy of its projection on the space $I_{h}(\sqrt{2})$ :

$$
\mathcal{E}_{h}\left(u_{h}\right) \leq M \mathcal{E}_{h}\left(\Upsilon_{h}^{1 / 3} u_{h}\right) \leq M \mathcal{E}_{h}\left(\Pi_{h}^{\sqrt{2}} u_{h}\right),
$$

i.e. $\left(u_{h}, u_{h}^{\prime}\right) \in K_{h}^{M}(\gamma)$ with $\gamma=\sqrt{2}$.

The following theorem gives us the property of uniform boundary observability for the solutions $u_{h}$ of system (1.6) with initial data $\left(u_{h}^{0}, u_{h}^{1}\right) \in V^{h} \times V^{h}$. This theorem is in fact a consequence of Theorem 2.1. estimate (3.5) and the well-known results on observability in classes of the form $I_{h}(\gamma)$ from [31] mentioned above.

Theorem 3.1. Let $T>4 \sqrt{2}$. There exists a constant $C(T)$ such that

$$
\mathcal{E}_{h}\left(u_{h}\right) \leq C(T) \int_{0}^{T} \int_{\Gamma_{0 h}}\left|\partial_{n}^{h} u_{h}\right|^{2} d \Gamma_{0 h} d t
$$

for all solutions $u_{h}$ of (1.6) with $\left(u_{h}^{0}, u_{h}^{1}\right) \in V^{h} \times V^{h}$, uniformly in $h>0, V^{h}$ being the class of two-grid data obtained with grids of mesh-size ratio $1 / 3$.

Remark 3.1. The time $T>4 \sqrt{2}$ corresponds to the class of solutions belonging to $I_{h}(\sqrt{2})$, the smallest class $I_{h}$ that contains $J_{h}(1 / 3)$, as obtained in [31]. Indeed, in view of 2.5 the known observability time for the above class of solutions is given by $T(\sqrt{2})=$ $4 \sqrt{2}$.

In fact, Theorem 3.1 would hold for all $T>T^{*}, T^{*}$ being the optimal time for uniform observability in the class $I_{h}(\sqrt{2})$. Very likely the estimate $T^{*}=4 \sqrt{2}$ given in [31] is not optimal. An analysis of the velocity of propagation of the associated bicharacteristic rays shows that, according to [32], the expected minimal time $T^{*}$ should be

$$
T^{*}=\frac{2 \sqrt{2}}{\cos (\pi / 6)}=\frac{4 \sqrt{2}}{\sqrt{3}} .
$$



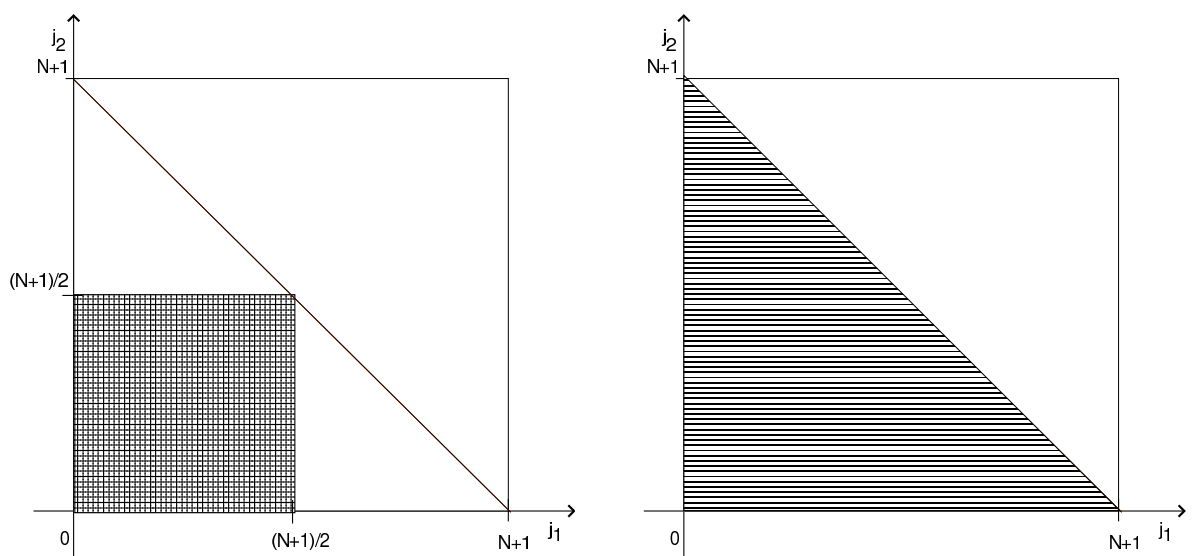

Fig. 6. Left: the black area represents the frequencies involved in $J_{h}(1 / 2)$; right: the dashed area represents the frequencies involved in $I_{h}(2)$.

Although the uniform observability inequality in the class $I_{h}(\sqrt{2})$ for all $T>T^{*}$ with $T^{*}$ as in 3.7 is very likely to hold, as far as we know, it has not been rigorously proved so far. Thus, improving the optimal time in Theorem 3.1 from $T>4 \sqrt{2}$ to $T>T^{*}$ as in 3.7 is an open problem. This improvement would also automatically lead to an improvement of the minimal time in Theorem 3.1

Remark 3.2. We could apply the same two-grid algorithm with grids of mesh-size ratio $1 / 2$, i.e. $G^{h}$ and $G^{2 h}$. In this case we would get, for some constant $C$, independent of $h$,

$$
\mathcal{E}_{h}\left(u_{h}\right) \leq C \mathcal{E}_{h}\left(\Upsilon_{h}^{1 / 2} u_{h}\right) \leq C \mathcal{E}_{h}\left(\Pi_{h}^{2} u_{h}\right)
$$

for all solutions $u_{h}$ obtained by this two-grid method. Indeed, the smallest $\gamma$ such that $I_{h}(\gamma)$ contains all the frequencies $\omega_{\mathbf{j}}(h)$ with $\|\mathbf{j}\|_{\infty} \leq(N+1) / 2$ is $\gamma=2$. Unfortunately, as we pointed out before, inequality (2.9) does not hold in the class $I_{h}(2)$. This is why we have chosen the ratio between the fine and coarse grids in the two-grid method to be $1 / 3$. This will guarantee that the two hypotheses 2.8 and $(2.9)$ are satisfied.

Remark 3.3. The method also works for mesh-size ratio $1 / p$ with $p \geq 3$. In this case,

$$
J_{h}\left(\frac{1}{p}\right) \subset I_{h}\left(2 \sqrt{2} \sin \left(\frac{\pi}{2 p}\right)\right)
$$

and thus the observability time given by Theorem 3.1 is

$$
T\left(2 \sqrt{2} \sin \left(\frac{\pi}{2 p}\right)\right)=\frac{2 \sqrt{2}}{\cos (\pi / p)} .
$$

Remark 3.4. The two-grid method proposed here always has a mesh-ratio of the form $1 / p$. The same two-grid algorithm makes sense for ratios $m / n$ with $m<n$. One could 
expect the uniform observability to hold in 1-d for any mesh-ratio $m / n<1$, in the multidimensional case, when $m / n<1 / 2$. But, by now, these are open problems. As we shall see, the only difficulty in doing that is to prove the following estimate for the functions $u_{h}^{0}$ belonging to $\Pi_{h}^{n / m h} \mathcal{G}_{h}$ :

$$
\left\|u_{h}^{0}\right\|_{s, h} \leq C(m / n, s)\left\|\Upsilon_{h}^{m / n} u_{h}^{0}\right\|_{s, h}, \quad s \in\{0,1\} .
$$

Proof of Theorem 3.1 As we shall see, Theorem 3.1 is an easy consequence of Theorem 2.1. Let $u_{h}$ be the solution of system (1.6) with initial data $\left(u_{h}^{0}, u_{h}^{1}\right) \in V^{h} \times V^{h}$. Using the inclusion $J_{h}(1 / 3) \subset I_{h}(\sqrt{2})$ we obtain

$$
\mathcal{E}_{h}\left(\Upsilon_{h}^{1 / 3} u_{h}\right) \leq \mathcal{E}_{h}\left(\Pi_{h}^{\sqrt{2}} u_{h}\right) .
$$

To apply Theorem 2.1 with $\gamma=\sqrt{2}$ it remains to prove 3.2, i.e. (3.3) and 3.4.

We make use of the following lemma, which will be proved in Appendix B.

Lemma 3.1. Let $p \geq 2$ and $V^{h}=\Pi_{h}^{p h}\left(\mathcal{G}_{p h}\right)$. For any $s \in[0,2]$ there exists a positive constant $C(p, s)$ such that

$$
\|v\|_{s, h} \leq C(p, s)\left\|\Upsilon_{h}^{1 / p} v\right\|_{s, h}, \quad 0 \leq s \leq 2,
$$

for any $v \in V^{h}$.

Applying this lemma with $p=3$ to $u_{h}^{0} \in V^{h}$ and $u_{h}^{1} \in V^{h}$ we get the existence of a positive constant $M=\max \{C(3,0), C(3,1)\}^{2}$ such that

$$
\left\|u_{h}^{0}\right\|_{1, h}^{2} \leq M\left\|\Upsilon_{h}^{1 / 3} u_{h}^{0}\right\|_{1, h}^{2} \quad \text { and } \quad\left\|u_{h}^{1}\right\|_{0, h}^{2} \leq M\left\|\Upsilon_{h}^{1 / 3} u_{h}^{1}\right\|_{0, h}^{2} .
$$

This proves [3.2) and finishes the proof of Theorem 3.1

\section{Proof of Theorem 2.1}

First of all we introduce the projectors $P_{k}$ that we shall use. Let $P \in C_{c}^{\infty}(\mathbb{R})$ and $c>1$. For any $f \in L^{1}(\mathbb{R})$ and $k \geq 0$ we define the projection $P_{k} f$ as follows:

$$
\left(P_{k} f\right)(t)=\int_{\mathbb{R}_{\tau}} \int_{\mathbb{R}_{s}} P\left(c^{-k} \tau\right) f(s) e^{i(t-s) \tau} d s d \tau, \quad t \in \mathbb{R} .
$$

In view of 2.6, for any $T>T(\gamma)$ there exist two positive constants $\delta$ and $\epsilon$ such that

$$
\mathcal{E}_{h}\left(v_{h}\right) \leq C(T, \gamma, \epsilon, \delta) \int_{2 \delta}^{T-2 \delta} \int_{\Gamma_{0 h}}\left|\partial_{h}^{h} v_{h}\right|^{2} d \Gamma_{0 h} d t
$$

for all $v_{h} \in I_{h}(\gamma+\epsilon)$. More precisely, using the continuity of the map $\gamma \mapsto T(\gamma)$ we obtain the existence of a small constant $\epsilon$ such that $T>T(\gamma+\epsilon)$. We then choose 
a positive $\delta$ such that $T-4 \delta>T(\gamma+\epsilon)$. Then the invariance under time translation guarantees that $(4.2)$ holds.

For $\epsilon$ satisfying 4.2 let us choose positive constants $a, b, c$ and $\mu$ satisfying

$$
1<c<\frac{b-\mu}{a+\mu} \text { and } \frac{b}{a+\mu}<\frac{\gamma+\epsilon}{\gamma} .
$$

Let $F \in C_{c}^{\infty}(\mathbb{R})$ be supported in $(a, b)$ with $0 \leq F \leq 1$ and $F \equiv 1$ in $[a+\mu, b-\mu]$. Set $P(\tau)=F(\tau)+F(-\tau)$ and then consider $P_{k}$ as in 4.1).

In view of 2.3 the Fourier transform of $u_{h}$, in the $t$ variable, reads

$$
\widehat{u}_{h}(\tau)=\sum_{\mathbf{j} \in \Lambda_{N}}\left[\delta\left(\tau-\omega_{\mathbf{j}}(h)\right) \widehat{u}_{\mathbf{j}+}^{h}+\delta\left(\tau+\omega_{\mathbf{j}}(h)\right) \widehat{u}_{\mathbf{j}-}^{h}\right] \varphi_{h}^{\mathbf{j}} .
$$

Therefore, the projector $P_{k} u_{h}$ is given by

$$
P_{k} u_{h}(t)=\sum_{\mathbf{j} \in \Lambda_{N}} F\left(c^{-k} \omega_{\mathbf{j}}(h)\right)\left[e^{i t \omega_{\mathbf{j}}(h)} \widehat{u}_{\mathbf{j}+}^{h}+e^{-i t \omega_{\mathbf{j}}(h)} \widehat{u}_{\mathbf{j}-}^{h}\right] \varphi_{h}^{\mathbf{j}}
$$

and its energy satisfies

$$
\mathcal{E}_{h}\left(P_{k} u_{h}\right)=\sum_{\mathbf{j} \in \Lambda_{N}} F^{2}\left(c^{-k} \omega_{\mathbf{j}}(h)\right) \omega_{\mathbf{j}}^{2}(h)\left(\left|\widehat{u}_{\mathbf{j}+}^{h}\right|^{2}+\left|\widehat{u}_{\mathbf{j}-}^{h}\right|^{2}\right) .
$$

Conditions 4.3 guarantee the existence of an index $k_{h}$ such that $\left\{P_{k} u_{h}\right\}_{k=0}^{k_{h}}$ covers all the frequencies occurring in the representation of $\Pi_{h}^{\gamma} u_{h}$, and all these projections belong to $I_{h}(\gamma+\epsilon)$.

Step I. Sketch of the main steps. We first give the main ideas of the proof. We choose $k_{h}$ as above and $k_{0} \leq k_{h}, k_{0}$ independent of $h$, such that $\left\{P_{k} u_{h}\right\}_{k=k_{0}}^{k_{h}}$ covers, except possibly for a finite number, all the frequencies occurring in $\Pi_{h}^{\gamma} u_{h}$, the projection of $u_{h}$ on the space $I_{h}(\gamma)$ defined in 2.4:

$$
\Pi_{h}^{\gamma} u_{h}=\frac{1}{2} \sum_{\omega_{\mathbf{j}}(h) \leq \gamma / h}\left[e^{i t \omega_{\mathbf{j}}(h)} \widehat{u}_{\mathbf{j}+}^{h}+e^{-i t \omega_{\mathbf{j}}(h)} \widehat{u}_{\mathbf{j}-}^{h}\right] \varphi_{h}^{\mathbf{j}} .
$$

The precise value of $k_{0}$ and $k_{h}$ will be specified later.

Our hypothesis on the initial data $\left(u_{h}^{0}, u_{h}^{1}\right) \in K_{\gamma}^{h}(M)$ guarantees (see 2.7) for the definition of the spaces $\left.K_{\gamma}^{h}(M)\right)$ that the total energy $u_{h}$ is controlled by the energy of $\Pi_{h}^{\gamma} u_{h}$ :

$$
\mathcal{E}_{h}\left(u_{h}\right) \leq M \mathcal{E}_{h}\left(\Pi_{h}^{\gamma} u_{h}\right)
$$

First, we will prove that

$$
\mathcal{E}_{h}\left(\Pi_{h}^{\gamma} u_{h}\right) \leq \sum_{k=k_{0}}^{k_{h}} \mathcal{E}_{h}\left(P_{k} u_{h}\right)+L O T
$$


where $L O T$ is a lower order term involving only a fixed number of Fourier components. In particular, LOT is compact in the sense that weak convergence in the energy space allows passing to the limit in it. In what follows, the precise value of LOT will change from line to line, but it will always denote a term involving a finite number of Fourier components, with the compactness property.

Next we use the fact that each projection $P_{k} u_{h}, k_{0} \leq k \leq k_{h}$, belongs to the class $I_{h}(\gamma+\epsilon)$, and consequently, according to 4.2 , satisfies the observability inequality:

$$
\mathcal{E}_{h}\left(P_{k} u_{h}\right) \leq C(T, \gamma, \delta, \epsilon) \int_{2 \delta}^{T-2 \delta} \int_{\Gamma_{0 h}}\left|\partial_{n}^{h} P_{k} u_{h}\right|^{2} d \Gamma_{0 h} d t
$$

Thus, combining (4.7) and 4.8) we obtain the estimate

$$
\mathcal{E}_{h}\left(\Pi_{h}^{\gamma} u_{h}\right) \leq C(T, \gamma, \delta, \epsilon) \sum_{k=k_{0}}^{k_{h}} \int_{2 \delta}^{T-2 \delta} \int_{\Gamma_{0 h}}\left|\partial_{n}^{h} P_{k} u_{h}\right|^{2} d \Gamma_{0 h} d t+L O T .
$$

Using ideas developed in [15] and [3] one can estimate the right hand side sum in terms of the energy of $u_{h}$ measured on $\Gamma_{0 h}$. More precisely, we will prove the existence of constants $C(P, c)$ and $C(\epsilon, \delta, T)$ such that

$$
\begin{aligned}
& \sum_{k \geq k_{0}} \int_{2 \delta}^{T-2 \delta} \int_{\Gamma_{h}}\left|\partial_{n}^{h} P_{k} u_{h}\right|^{2} d \Gamma_{h} d t \\
& \quad \leq C(P, c) \int_{0}^{T} \int_{\Gamma_{0 h}}\left|\partial_{n}^{h} u_{h}\right|^{2} d \Gamma_{h} d t+\frac{C(\epsilon, \delta, T)}{c^{2 k_{0}}} \mathcal{E}_{h}\left(u_{h}\right)
\end{aligned}
$$

for any $k_{0} \geq 0$ and $u_{h}$ solving (1.6), uniformly in $h>0$. Then combining (4.6), (4.9) and 4.10) gives

$$
\begin{aligned}
\mathcal{E}_{h}\left(u_{h}\right) \leq & C(T, P, \gamma, \delta, \epsilon, c) \int_{0}^{T} \int_{\Gamma_{0 h}}\left|\partial_{n}^{h} u_{h}\right|^{2} d \Gamma_{0 h} d t \\
& +\frac{C(\epsilon, \delta, T)}{c^{2 k_{0}}} \mathcal{E}_{h}\left(u_{h}\right)+L O T .
\end{aligned}
$$

If we choose $h$ small and $k_{0}$ sufficiently large, but still independent of $h$, the energy term from the right side may be absorbed and then we obtain

$$
\mathcal{E}_{h}\left(u_{h}\right) \leq C(T, P, \gamma, \delta, \epsilon, c) \int_{0}^{T} \int_{\Gamma_{0 h}}\left|\partial_{n}^{h} u_{h}\right|^{2} d \Gamma_{0 h} d t+L O T .
$$

Finally, classical arguments of compactness-uniqueness allow us to get rid of the lower order term. For a complete development of this argument we refer to [31].

In the following we give the details of the proofs of the above steps.

Step II. Upper bounds of $\mathcal{E}_{h}\left(\Pi_{h}^{\gamma} u_{h}\right)$ in terms of $\left\{\mathcal{E}_{h}\left(P_{k} u_{h}\right)\right\}_{k \geq 0}$. Let us choose a positive integer $k_{h}$ such that

$$
c^{k_{h}}(a+\mu) \leq \gamma / h<c^{k_{h}+1}(a+\mu) .
$$


The choice of $k_{h}$ is always possible for $h$ small enough. Also let us fix a positive integer $k_{0} \leq k_{h}$ independent of $h$. Its precise value will be chosen later. From $c<(b-\mu) /(a+\mu)$ (see 4.3) we obtain

$$
c^{k_{h}}(a+\mu) \leq \gamma / h \leq c^{k_{h}+1}(a+\mu) \leq c^{k_{h}}(b-\mu) .
$$

Then any frequency $\omega_{\mathbf{j}}(h)$ belonging to $\left[(a+\mu) c^{k_{0}}, \gamma / h\right]$ is contained in at least one interval of the form $\left[c^{k}(a+\mu), c^{k}(b-\mu)\right]$ with $k_{0} \leq k \leq k_{h}$ where $F\left(c^{-k} \cdot\right) \equiv 1$. Thus for any frequency $\omega_{\mathbf{j}}(h) \in\left[(a+\mu) c^{k_{0}}, \gamma / h\right]$ we have

$$
1 \leq \sum_{k=k_{0}}^{k_{h}} F\left(c^{-k} \omega_{\mathbf{j}}(h)\right)^{2}
$$

In view of 4.5 and 4.14 the energy of $\Pi_{h}^{\gamma} u_{h}$, excepting a lower order term involving a finite number of Fourier components only, can be bounded above by the energy of all the projections $\left(P_{k} u_{h}\right)_{k=k_{0}}^{k_{h}}$ :

$$
\begin{aligned}
\mathcal{E}_{h}\left(\Pi_{h}^{\gamma} u_{h}\right) \leq & c^{2 k_{0}}(a+\mu)^{2} \sum_{\omega_{\mathbf{j}}(h)<(a+\mu) c^{k_{0}}}\left(\left|\widehat{u}_{\mathbf{j}+}^{h}\right|^{2}+\left|\widehat{u}_{\mathbf{j}-}^{h}\right|^{2}\right) \\
& +\sum_{k=k_{0}}^{k_{h}} \sum_{\mathbf{j} \in \Lambda_{N}} F^{2}\left(c^{-k} \omega_{\mathbf{j}}(h)\right) \omega_{\mathbf{j}}^{2}(h)\left(\left|\widehat{u}_{\mathbf{j}+}^{h}\right|^{2}+\left|\widehat{u}_{\mathbf{j}-}^{h}\right|^{2}\right) \\
= & C\left(a, k_{0}, \mu\right) \sum_{\omega_{\mathbf{j}}(h)<(a+\mu) c^{k_{0}}}\left(\left|\widehat{u}_{\mathbf{j}+}^{h}\right|^{2}+\left|\widehat{u}_{\mathbf{j}-}^{h}\right|^{2}\right)+\sum_{k=k_{0}}^{k_{h}} \mathcal{E}_{h}\left(P_{k} u_{h}\right) .
\end{aligned}
$$

Step III. Observability inequalities for the projections $P_{k} u_{h}, k \leq k_{h}$. The next step is to apply the observability inequality (4.2) to each projection $P_{k} u_{h}, k \leq k_{h}$. We show that each of them belongs to the class $I_{h}(\gamma+\epsilon)$ where (4.2) holds. We remark that the projector $P_{k} u_{h}$ contains only the frequencies $\omega_{\mathbf{j}}(h) \in\left(c^{k} a, c^{k} b\right)$. In view of 4.13 any frequency $\omega_{\mathbf{j}}(h)$ involved in the decomposition of $P_{k} u_{h}, k \leq k_{h}$, satisfies

$$
\omega_{\mathbf{j}}(h)<c^{k_{h}} b \leq \frac{\gamma b}{h(a+\mu)}<\frac{\gamma+\epsilon}{h},
$$

which shows that $P_{k} u_{h} \in I_{h}(\gamma+\epsilon)$. Then for any $k \leq k_{h}$,

$$
\mathcal{E}_{h}\left(P_{k} u_{h}\right) \leq C(T, \delta, \epsilon, \gamma) \int_{2 \delta}^{T-2 \delta} \int_{\Gamma_{0 h}}\left|\partial_{n}^{h}\left(P_{k} u_{h}\right)\right|^{2} d \Gamma_{0 h} d t .
$$

Using (4.15) and the above inequalities we obtain

$$
\begin{aligned}
\mathcal{E}_{h}\left(\Pi_{h}^{\gamma} u_{h}\right) \leq & C(T, \gamma, \delta, \epsilon) \sum_{k=k_{0}}^{k_{h}} \int_{2 \delta}^{T-2 \delta} \int_{\Gamma_{0 h}}\left|\partial_{n}^{h}\left(P_{k} u_{h}\right)\right|^{2} d \Gamma_{0 h} d t \\
& +C\left(a, k_{0}, \mu\right) \sum_{\omega_{\mathbf{j}}(h)<(a+\mu) c^{k_{0}}}\left[\left|\widehat{u}_{\mathbf{j}+}^{h}\right|^{2}+\left|\widehat{u}_{\mathbf{j}-}^{h}\right|^{2}\right] .
\end{aligned}
$$


It remains to prove (4.10). This inequality implies (4.11) and (4.12), which finishes the proof.

The key point is the following lemma which will be proved in Appendix A

Lemma 4.1. Let $\mu$ be a Borel measure, $\Omega$ a $\mu$-measurable set such that $\mu(\Omega)<\infty$, $P \in C_{c}^{\infty}(\mathbb{R}), c>1$ and $1 \leq p \leq \infty$. Set $X=L^{p}(\Omega, d \mu)$ and $P_{k}$ as in 4 (4.1). For any positive $T$ and $\delta<T / 4$ there are positive constants $C(P, c)$ and $C(\delta, T, P)$ such that

$$
\sum_{k \geq k_{0}} \int_{2 \delta}^{T-2 \delta}\left\|P_{k} w\right\|_{X}^{2} d t \leq C(P, c) \int_{0}^{T}\|w\|_{X}^{2} d t+\frac{C(\delta, T, P)}{c^{2 k_{0}}} \sup _{l \in \mathbb{Z}}\|w\|_{L^{2}((l T,(l+1) T), X)}^{2}
$$

for all positive integers $k_{0}$ and $w \in L_{\text {loc }}^{2}(\mathbb{R}, X)$.

We now apply Lemma 4.1 with $X=l^{2}\left(\Gamma_{0 h}\right)$ and $w=\partial_{n}^{h} u_{h}$. Since $P_{k}\left(\partial_{n}^{h} u_{h}\right)=$ $\partial_{n}^{h}\left(P_{k} u_{h}\right)$, we obtain the existence of a constant $C(\delta, T, P)$ such that

$$
\begin{aligned}
\sum_{k \geq k_{0}} \int_{2 \delta}^{T-2 \delta} \int_{\Gamma_{0 h}}\left|\partial_{n}^{h} P_{k} u_{h}(t)\right|^{2} d \Gamma_{0 h} d t & \leq C(P, c) \int_{0}^{T} \int_{\Gamma_{0 h}}\left|\partial_{n}^{h} u_{h}(t)\right|^{2} d \Gamma_{0 h} d t \\
+ & \frac{C(\delta, T, P)}{c^{2 k_{0}}} \sup _{l \in \mathbb{Z}} \int_{l T}^{(l+1) T} \int_{\Gamma_{0 h}}\left|\partial_{n}^{h} u_{h}(t)\right|^{2} d \Gamma_{0 h} d t .
\end{aligned}
$$

At this point we apply the so-called "direct inequality" (2.10), which holds for all solutions $u_{h}$ of system (1.6). Thus, a translation in time in 2.10$)$ together with the conservation of energy shows that

$$
\sup _{l \in \mathbb{Z}} \int_{l T}^{(l+1) T} \int_{\Gamma_{0 h}}\left|\partial_{n}^{h} u_{h}(t)\right|^{2} d \Gamma_{0 h} d t \leq C(T) \mathcal{E}_{h}\left(u_{h}\right),
$$

and so 4.10 holds.

\section{Construction of the control}

In this section we introduce a numerical approximation for the HUM control $v$ of the continuous wave equation (1.1) based on the two-grid method.

First, we define a restriction operator which carries any function of $\mathcal{G}_{h}$ to $\mathcal{G}_{3 h}$. The most natural way is to define it as the formal adjoint of the $\Pi_{h}^{3 h}$ operator:

$$
\left(\psi, \Pi_{h}^{3 h} \phi\right)_{h}=\left(\Pi_{h}^{3 h, *} \psi, \phi\right)_{3 h}, \quad \forall \phi \in \mathcal{G}_{3 h} .
$$

To obtain the control $v_{h}$ in (1.5) that is intended to approximate the control of (1.1), it would be rather natural to approximate the initial data $\left(y^{0}, y^{1}\right)$ by $\left(y_{h}^{0}, y_{h}^{1}\right)$ and take the corresponding controls $v_{h}$. But this has to be done carefully taking into account the high frequency pathologies. In fact, not all the approximation of the initial data has to be done carefully but also the final requirement $(1.2)$ has to be relaxed conveniently. To do this we shall consider controls $v_{h}$ for which $\Pi_{h}^{3 h, *} y_{h}$, the projection of solutions over the coarse grid $G^{4 h}$, vanishes at the time $t=T$. 
Theorem 5.1. Let $T>4 \sqrt{2}$. There exists a constant $C(T)$ such that for any $h>0$ and $\left(y_{h}^{0}, y_{h}^{1}\right)$, there exists a function $v_{h}$ satisfying

$$
\left\|v_{h}\right\|_{L^{2}\left((0, T) \times \Gamma_{0 h}\right)}^{2} \leq C(T)\left(\left\|y_{h}^{0}\right\|_{0, h}^{2}+\left\|y_{h}^{1}\right\|_{-1, h}^{2}\right)
$$

such that the solution $u_{h}$ of system (1.5) with $\left(y_{h}^{0}, y_{h}^{1}\right)$ as initial data and $v^{h}$ acting as control satisfies

$$
\boldsymbol{\Pi}_{h}^{3 h, *} y_{h}(T)=\Pi_{h}^{3 h, *} y_{h}^{\prime}(T)=0 .
$$

In order to construct the function $v_{h}$ we need some notations and preliminary results. We define the duality product between $L^{2}(\Omega) \times H^{-1}(\Omega)$ and $H_{0}^{1}(\Omega) \times L^{2}(\Omega)$ by

$$
\left\langle\left(\varphi^{0}, \varphi^{1}\right),\left(u^{0}, u^{1}\right)\right\rangle=\left(\varphi^{1}, u^{0}\right)_{-1,1}-\left(\varphi^{0}, u^{1}\right) .
$$

Also for the discrete spaces $\mathcal{H}_{h}^{0}\left(\Omega_{h}\right) \times \mathcal{H}_{h}^{-1}\left(\Omega_{h}\right)$ and $\mathcal{H}_{h}^{1}\left(\Omega_{h}\right) \times \mathcal{H}_{h}^{0}\left(\Omega_{h}\right)$ we introduce a similar duality product

$$
\left\langle\left(\varphi^{0}, \varphi^{1}\right),\left(u^{0}, u^{1}\right)\right\rangle_{h}=\left(\varphi^{1}, u^{0}\right)_{h}-\left(\varphi^{0}, u^{1}\right)_{h} .
$$

Let us introduce the adjoint discrete problem:

$$
\begin{cases}u_{h}^{\prime \prime}-\Delta_{h} u_{h}=0 & \text { in } \Omega_{h} \times(0, T), \\ u_{h}(t)=0 & \text { on } \Gamma_{h} \times(0, T), \\ u_{h}(T)=u_{h}^{0}, \partial_{t} u_{h}(T)=u_{h}^{1} & \text { in } \Omega_{h} .\end{cases}
$$

Note that the system (5.3) can be transformed into 1.6$)$ by reversing time $(t \mapsto T-t)$. Thus, all the previous estimates on (1.6) apply to $(5.3)$ too.

Following the same steps as in the continuous case, i.e. multiplying the control problem (1.5) by solutions of the adjoint problem 5.3) and integrating (summing) by parts, we obtain the following result for the solutions of system 1.5 :

Lemma 5.1. Let $y_{h}$ be a solution of system (1.5). Then

$$
\int_{0}^{T} \int_{\Gamma_{0 h}} v_{h}(t) \partial_{n}^{h} u_{h}(t) d \Gamma_{1 h} d t+\left.\left\langle\left(y_{h}, y_{h}^{\prime}\right),\left(u_{h}, u_{h}^{\prime}\right)\right\rangle_{h}\right|_{0} ^{T}=0
$$

for all solutions $u_{h}$ of the adjoint problem [5.3).

Proof. Multiplying (1.5) and (5.3) by $u_{h}$, respectively $y_{h}$, integrating on $[0, T]$ and summing over $\Omega_{h}$ yields

$$
\int_{0}^{T} \int_{\Omega_{h}}\left(y_{h}^{\prime \prime} u_{h}-u_{h}^{\prime \prime} y_{h}\right) d \Omega_{h} d t=\int_{0}^{T} \int_{\Omega_{h}}\left[\left(\Delta_{h} y_{h}\right) u_{h}-\left(\Delta_{h} u_{h}\right) y_{h}\right] d \Omega_{h} d t .
$$

Integration by parts on the left hand side term gives

$$
\begin{aligned}
\int_{\Omega_{h}} \int_{0}^{T}\left(y_{h}^{\prime \prime} u_{h}-u_{h}^{\prime \prime} y_{h}\right) d t d \Omega_{h} & =\int_{\Omega_{h}}\left(\left.y_{h}^{\prime} u_{h}\right|_{0} ^{T}-\left.u_{h}^{\prime} y_{h}\right|_{0} ^{T}\right) d \Omega_{h} \\
& =\left.\left\langle\left(y_{h}, y_{h}^{\prime}\right),\left(u_{h}, u_{h}^{\prime}\right)\right\rangle_{h}\right|_{0} ^{T} .
\end{aligned}
$$


For the second term of (5.5) we have

$$
\begin{aligned}
& \int_{0}^{T} \int_{\Omega_{h}}\left[\left(\Delta_{h} y_{h}\right) u_{h}-\left(\Delta_{h} u_{h}\right) y_{h}\right] d \Omega_{h} d t \\
& =\sum_{i, j=1}^{N}\left[\left(y_{i-1, j}+y_{i+1, j}\right) u_{i, j}-\left(u_{i-1, j}+u_{i+1, j}\right) y_{i, j}\right] \\
& \quad+\sum_{i, j=1}^{N}\left[\left(y_{i, j-1}+y_{i, j+1}\right) u_{i, j}-\left(u_{i, j-1}+u_{i, j+1}\right) y_{i, j}\right] \\
& =\sum_{j=1}^{N}\left(y_{0, j} u_{1, j}+y_{N+1, j} u_{N, j}\right)+\sum_{i=1}^{N}\left(y_{i, 0} u_{i, 1}+y_{i, N+1} u_{i, N}\right) \\
& =\sum_{j=1}^{N} y_{N+1, j} u_{N, j}+\sum_{i=1}^{N} y_{i, N+1} u_{i, N}=-\int_{0}^{T} \int_{\Gamma_{0 h}} v_{h}(t) \partial_{n}^{h} u_{h}(t) d t d \Gamma_{1 h} .
\end{aligned}
$$

Identities 5.6 and 5.7 prove 5.4.

Proof of Theorem 5.1. Step I. Construction of $v_{h}$. First, using variational methods we will prove the existence of a function $v_{h}$ such that

$$
\int_{0}^{T} \int_{\Gamma_{0 h}} v_{h}(t) \partial_{n}^{h} u_{h}(t) d \Gamma_{0 h} d t+\left\langle\left(y_{h}^{0}, y_{h}^{1}\right),\left(u_{h}(0), u_{h}^{\prime}(0)\right)\right\rangle_{h}=0
$$

for all solutions $u_{h}$ of the adjoint problem 5.3 with final state $\left(u_{h}^{0}, u_{h}^{1}\right) \in V^{h} \times V^{h}$. This is equivalent to (5.2) in view of (5.4).

To do this we consider the space $\mathcal{F}_{h}=V^{h} \times V^{h}$ endowed with the norm

$$
\left\|\left(u_{h}^{0}, u_{h}^{1}\right)\right\|_{\mathcal{F}_{h}}=\left(\left\|u_{h}^{0}\right\|_{1, h}^{2}+\left\|u_{h}^{1}\right\|_{0, h}^{2}\right)^{1 / 2}
$$

and the functional $\mathcal{J}_{h}: \mathcal{F}_{h} \rightarrow \mathbb{R}$ defined by

$$
\mathcal{J}_{h}\left(\left(u_{h}^{0}, u_{h}^{1}\right)\right)=\frac{1}{2} \int_{0}^{T} \int_{\Gamma_{0 h}}\left|\partial_{n}^{h} u_{h}\right|^{2} d \Gamma_{0 h} d t+\left\langle\left(y_{h}^{0}, y_{h}^{1}\right),\left(u_{h}(0), u_{h}^{\prime}(0)\right)\right\rangle_{h}
$$

where $u_{h}$ is the solution of the adjoint problem 5.3 with final state $\left(u_{h}^{0}, u_{h}^{1}\right)$. To construct the control $v_{h}$ satisfying the relaxed controllability condition 5.8 for all $\left(u_{h}^{0}, u_{h}^{1}\right) \in$ $V^{h} \times V^{h}$ it is sufficient to minimize $\mathcal{J}_{h}$ over $\mathcal{F}_{h}$.

In order to apply the fundamental theorem of the calculus of variations, guaranteeing the existence of a minimizer for $\mathcal{J}_{h}$, we prove that the functional $\mathcal{J}_{h}$ restricted to $\mathcal{F}_{h}$, which is convex, is also continuous and uniformly coercive (with respect to the parameter $h$ ).

The linear term on the right side of (5.9) satisfies

$$
\left|\left\langle\left(y_{h}^{0}, y_{h}^{1}\right),\left(u_{h}(0), u_{h}^{\prime}(0)\right)\right\rangle_{h}\right| \leq\left(\left\|y_{h}^{1}\right\|_{-1, h}+\left\|y_{h}^{0}\right\|_{0, h}\right)\left\|\left(u_{h}(0), u_{h}^{\prime}(0)\right)\right\|_{\mathcal{F}_{h}} .
$$


Using the direct inequality 2.10 and the conservation of the energy $\mathcal{E}_{h}\left(u_{h}\right)$ we get

$$
\left|\mathcal{J}_{h}\left(\left(u_{h}^{0}, u_{h}^{1}\right)\right)\right| \leq\left\|\left(u_{h}^{0}, u_{h}^{1}\right)\right\|_{\mathcal{F}_{h}}\left(C(T)\left\|\left(u_{h}^{0}, u_{h}^{1}\right)\right\|_{\mathcal{F}_{h}}+\left\|y_{h}^{1}\right\|_{-1, h}+\left\|y_{h}^{0}\right\|_{0, h}\right),
$$

which proves the continuity of the functional $\mathcal{J}_{h}$.

In view of the observability inequality 3.6 , for any $T>4 \sqrt{2}$, the functional $\mathcal{J}_{h}$ is uniformly (with respect to $h$ ) coercive on $\mathcal{F}_{h}$ :

$$
\left|\mathcal{J}_{h}\left(\left(u_{h}^{0}, u_{h}^{1}\right)\right)\right| \geq\left\|\left(u_{h}^{0}, u_{h}^{1}\right)\right\|_{\mathcal{F}_{h}}\left(C(T)\left\|\left(u_{h}^{0}, u_{h}^{1}\right)\right\|_{\mathcal{F}_{h}}-\left\|y_{h}^{1}\right\|_{-1, h}-\left\|y_{h}^{0}\right\|_{0, h}\right)
$$

for all $\left(u_{h}^{0}, u_{h}^{1}\right) \in \mathcal{F}_{h}$, where $C(T)$ is the constant obtained in 3.6 .

Applying the fundamental theorem of the calculus of variations we obtain the existence of a minimizer $\left(u_{h}^{0, *}, u_{h}^{1, *}\right) \in \mathcal{F}_{h}$ such that

$$
\mathcal{J}_{h}\left(\left(u_{h}^{0, *}, u_{h}^{1, *}\right)\right)=\min _{\left(\left(u_{h}^{0}, u_{h}^{1}\right)\right) \in \mathcal{F}_{h}} \mathcal{J}_{h}\left(\left(u_{h}^{0}, u_{h}^{1}\right)\right) .
$$

This implies that $\mathcal{J}_{h}^{\prime}$, the Gateaux derivative of $\mathcal{J}_{h}$, satisfies

$$
\mathcal{J}_{h}^{\prime}\left(\left(u_{h}^{0, *}, u_{h}^{1, *}\right)\right)\left(u_{h}^{0}, u_{h}^{1}\right)=0
$$

for all $\left(u_{h}^{0}, u_{h}^{1}\right) \in \mathcal{F}_{h}$, and that any $u_{h}^{*}$ solving 5.3 with final state $\left(u_{h}^{0, *}, u_{h}^{1, *}\right)$ satisfies

$$
\int_{0}^{T} \int_{\Gamma_{0 h}}\left(\partial_{n}^{h} u_{h}^{*}\right) \partial_{n}^{h} u(t) d \Gamma_{0 h} d t+\left\langle\left(y_{h}^{0}, y_{h}^{1}\right),\left(u_{h}(0), u_{h}^{\prime}(0)\right)\right\rangle_{h}=0
$$

for all $u_{h}$ solving the adjoint problem 5.3 with final state $\left(u_{h}^{0}, u_{h}^{1}\right) \in \mathcal{F}_{h}$.

We set

$$
v_{h}(t)=\partial_{n}^{h} u_{h}^{*}(t), \quad t \in[0, T],
$$

and then 5.8 holds.

Step II. Proof of property (5.2). In view of Lemma 5.1, the solution $y_{h}$ of system 1.5 with the above function $v_{h}$ acting as control on $\Gamma_{0 h}$ satisfies

$$
\left(y_{h}^{\prime}(T), u_{h}^{0}\right)_{h}-\left(y_{h}(T), u_{h}^{1}\right)_{h}=0
$$

for all $\left(u_{h}^{0}, u_{h}^{1}\right) \in V^{h} \times V^{h}$. As $V^{h}=\Pi_{h}^{3 h}\left(\mathcal{G}^{3 h}\right)$ we obtain

$$
\left(y_{h}(T), \boldsymbol{\Pi}_{h}^{3 h} w\right)_{h}=\left(y_{h}^{\prime}(T), \boldsymbol{\Pi}_{h}^{3 h} w\right)_{h}=0
$$

for all $w \in \mathcal{G}^{3 h}$. Then

$$
\left(\boldsymbol{\Pi}_{h}^{3 h, *} y_{h}(T), w\right)_{3 h}=\left(\boldsymbol{\Pi}_{h}^{3 h, *} y_{h}^{\prime}(T), w\right)_{3 h}=0
$$

for all $w \in \mathcal{G}^{3 h}$ and obviously (5.2) holds. 
Step III. Proof of estimate 5.1). Since $\left(u_{h}^{0, *}, \phi_{h}^{1, *}\right)$ is a minimizer of $\mathcal{J}_{h}$ we have $\mathcal{J}_{h}\left(\left(u_{h}^{0, *}, u_{h}^{1, *}\right)\right) \leq \mathcal{J}_{h}\left(\left(0_{h}, 0_{h}\right)\right)$, where $0_{h}$ is the function vanishing identically on the mesh $G_{h}$. Consequently,

$$
\int_{0}^{T} \int_{\Gamma_{0 h}}\left|\partial_{n}^{h} u_{h}^{*}\right|^{2} d \Gamma_{0 h} d t \leq\left(\left\|y_{h}^{1}\right\|_{-1, h}+\left\|y_{h}^{0}\right\|_{0, h}\right)\left(\left\|u_{h}^{0, *}\right\|_{1, h}+\left\|u_{h}^{1, *}\right\|_{0, h}\right) .
$$

Applying the observability inequality (3.6) to the solution $u_{h}^{*}$ we get

$$
\left\|u_{h}^{0, *}\right\|_{1, h}^{2}+\left\|u_{h}^{1, *}\right\|_{0, h}^{2} \leq C(T) \int_{0}^{T} \int_{\Gamma_{0 h}}\left|\partial_{n}^{h} u_{h}^{*}\right|^{2} d \Gamma_{0 h} d t .
$$

We then find that

$$
\left\|v_{h}\right\|_{L^{2}\left((0, T) \times \Gamma_{0 h}\right)}^{2}=\int_{0}^{T} \int_{\Gamma_{0 h}}\left|\partial_{n}^{h} u_{h}^{*}\right|^{2} d \Gamma_{0 h} d t \leq C(T)\left(\left\|y_{h}^{0}\right\|_{-1, h}+\left\|y_{h}^{1}\right\|_{0, h}\right)^{2}
$$

where the constant $C(T)$ is independent of $h$.

The proof is now complete.

\section{Convergence of the uncontrolled problem}

In this section, for the sake of completeness, we prove the convergence of the solutions of the uncontrolled problem (1.6). We also analyze the convergence of their normal derivatives towards the continuous one. First we introduce the interpolators needed in our analysis.

\subsection{Interpolators}

We denote by $\mathbf{P}_{h}^{1}$ the piecewise multi-linear and continuous interpolator on $\Omega$. We also consider the operators $\mathbf{P}_{h}^{s}$ defined for any $u \in \mathcal{H}_{h}^{s}(\Omega)$ by

$$
\mathbf{P}_{h}^{s} u_{h}=(-\Delta)^{-(s-1) / 2}\left(\mathbf{P}_{h}^{1}\left(-\Delta_{h}\right)^{(s-1) / 2} u_{h}\right) ;
$$

note that for any $s \in \mathbb{R}$, they continuously map $\mathcal{H}_{h}^{s}\left(\Omega_{h}\right)$ to $H^{s}(\Omega)$.

We will denote by $\nabla_{h}^{+}$the operator

$$
\left(\nabla_{h}^{+} u\right)_{j, k}=\left(\frac{u_{j+1, k}-u_{j, k}}{h}, \frac{u_{j, k+1}-u_{j, k}}{h}\right) .
$$

The representation of the operator $\mathbf{P}_{h}^{0}$ in the Fourier space shows that this operator is exactly the piecewise constant interpolator:

$$
\mathbf{P}_{h}^{0} u_{h}(x)=u_{j k}, \quad x \in((j-1 / 2) h,(j+1 / 2) h) \times((k-1 / 2) h,(k+1 / 2) h) .
$$


The operator $\mathbf{P}_{h}^{-1}$ satisfies

$$
\left\|\mathbf{P}_{h}^{-1} u_{h}\right\|_{H^{-1}(\Omega)}=\left\|\mathbf{P}_{h}^{1}\left(-\Delta_{h}\right)^{-1} u_{h}\right\|_{H_{0}^{1}(\Omega)}=\left\|\nabla_{h}^{+}\left(-\Delta_{h}\right)^{-1} u_{h}\right\|_{\mathcal{H}_{h}^{0}\left(\Omega_{h}\right)}=\left\|u_{h}\right\|_{\mathcal{H}_{h}^{-1}\left(\Omega_{h}\right)} .
$$

Also for any pair of functions $u_{h}$ and $w_{h}$ defined on $G^{h}$ and vanishing on $\Gamma_{h}$,

$$
\begin{aligned}
\int_{\Omega} \mathbf{P}_{h}^{0} u_{h} \mathbf{P}_{h}^{0} w_{h} & =\int_{\Omega_{h}} u_{h} w_{h} d \Omega_{h}=\int_{\Omega_{h}}\left(-\Delta_{h}\right)\left(-\Delta_{h}\right)^{-1} u_{h} w_{h} d \Omega_{h} \\
& =\int_{\Omega_{h}} \nabla_{h}\left(\left(-\Delta_{h}\right)^{-1} u_{h}\right) \cdot \nabla_{h} w_{h} d \Omega=\int_{\Omega} \nabla\left(\mathbf{P}_{h}^{1}\left(-\Delta_{h}\right)^{-1} u_{h}\right) \cdot \nabla\left(\mathbf{P}_{h}^{1} w_{h}\right) \\
& =\left\langle\mathbf{P}_{h}^{-1} u_{h}, \mathbf{P}_{h}^{1} w_{h}\right\rangle_{-1,1} .
\end{aligned}
$$

Lemma 6.1. For all $h>0$ and all sequences $u_{h}$,

$$
\left\|\mathbf{P}_{h}^{-1} u_{h}-\mathbf{P}_{h}^{0} u_{h}\right\|_{H^{-1}(\Omega)} \leq h\left\|u_{h}\right\|_{0, h} .
$$

Proof. By the definition of the operators $\mathbf{P}_{h}^{-1}$ and $\mathbf{P}_{h}^{0}$ we get

$$
(-\Delta)^{-1 / 2} \mathbf{P}_{h}^{0} u_{h}=\mathbf{P}_{h}^{1}\left(-\Delta_{h}\right)^{-1 / 2} u_{h}, \quad \mathbf{P}_{h}^{-1} u_{h}=(-\Delta)^{1 / 2} \mathbf{P}_{h}^{0}(-\Delta)^{-1 / 2} u_{h} .
$$

Thus we have

$$
\begin{aligned}
\left\|\mathbf{P}_{h}^{-1} u_{h}-\mathbf{P}_{h}^{0} u_{h}\right\|_{H^{-1}(\Omega)} & =\left\|(-\Delta)^{1 / 2} \mathbf{P}_{h}^{0}\left(-\Delta_{h}\right)^{-1 / 2} u_{h}-\mathbf{P}_{h}^{0} u_{h}\right\|_{H^{-1}(\Omega)} \\
& =\left\|\mathbf{P}_{h}^{0}\left(-\Delta_{h}\right)^{-1 / 2} u_{h}-(-\Delta)^{-1 / 2} \mathbf{P}_{h}^{0} u_{h}\right\|_{L^{2}(\Omega)} \\
& =\left\|\mathbf{P}_{h}^{0}\left(-\Delta_{h}\right)^{-1 / 2} u_{h}-\mathbf{P}_{h}^{1}\left(-\Delta_{h}\right)^{-1 / 2} u_{h}\right\|_{L^{2}(\Omega)}
\end{aligned}
$$

Since the two interpolators $\mathbf{P}_{h}^{0}$ and $\mathbf{P}_{h}^{1}$ satisfy (see [27, Th. 3.4.1, p. 88])

$$
\left\|\mathbf{P}_{h}^{0} u_{h}-\mathbf{P}_{h}^{1} u_{h}\right\|_{L^{2}(\Omega)} \leq h\left\|u_{h}\right\|_{\mathcal{H}_{h}^{1}(\Omega)}
$$

we obtain

$$
\left\|\mathbf{P}_{h}^{-1} u_{h}-\mathbf{P}_{h}^{0} u_{h}\right\|_{H^{-1}(\Omega)} \leq h\left\|\left(-\Delta_{h}\right)^{-1 / 2} u_{h}\right\|_{\mathcal{H}_{h}^{1}\left(\Omega_{h}\right)}=h\left\|u_{h}\right\|_{l^{2}\left(\Omega_{h}\right)},
$$

which finishes the proof.

\subsection{Convergence of the solutions}

The following propositions describe how a uniformly bounded family of solutions of (1.6) weakly converges (up to a subsequence) as $h \rightarrow 0$ to a solution of finite energy of the continuous wave equation (1.3).

Let us consider the family $\left\{u_{h}\right\}_{h>0}$ of solutions of $(1.6)$ and denote by $\mathbf{P}_{h}^{1} u_{h}$ their piecewise linear interpolator, which belongs to $H_{0}^{1}(\Omega)$ for all $0 \leq t \leq T$, just as the solution of the continuous problem does. 
Proposition 6.1. Let $\left\{u_{h}\right\}_{h>0}$ be a family of solutions of (5.3) depending on the parameter $h \rightarrow 0$, whose energies are uniformly bounded, i.e.

$$
E_{h}(0) \leq C, \quad \forall h>0 .
$$

Then there exists a solution $u \in C\left([0, T], H_{0}^{1}(\Omega)\right) \cap C^{1}\left([0, T], L^{2}(\Omega)\right)$ of problem 1.3 such that, by extracting a suitable subsequence $h \rightarrow 0$, we may guarantee that

$$
\begin{aligned}
& \mathbf{P}_{h}^{1} u_{h} \rightarrow u \quad \text { in } L^{2}\left([0, T], H_{0}^{1}(\Omega)\right), \\
& \mathbf{P}_{h}^{0} u_{h}^{\prime} \rightarrow u^{\prime} \quad \text { in } L^{2}\left([0, T], L^{2}(\Omega)\right) .
\end{aligned}
$$

Moreover, if the family $\left\{u_{h}\right\}_{h>0}$ is such that $\mathbf{P}_{h}^{1} u_{h}(0) \rightarrow u^{0}$ in $H_{0}^{1}(\Omega)$ and $\mathbf{P}_{h}^{0} u_{h}^{\prime}(0) \rightarrow u^{1}$ in $L^{2}(\Omega)$ for some $\left(u^{0}, u^{1}\right) \in H^{1}(\Omega) \times L^{2}(\Omega)$ then all the above convergences hold in the corresponding strong topologies.

Proof of Proposition 6.1. Step I. Weak convergence. In view of the uniform bound (6.3) and the conservation of energy we deduce that

$$
\left\{\begin{array}{l}
\mathbf{P}_{h}^{1} u_{h} \text { is uniformly bounded in } W^{1, \infty}\left((0, T), L^{2}(\Omega)\right), \\
\mathbf{P}_{h}^{0} u_{h} \text { is uniformly bounded in } W^{1, \infty}\left((0, T), L^{2}(\Omega)\right)
\end{array}\right.
$$

Using that

$$
\left\|\mathbf{P}_{h}^{1} u_{h}-\mathbf{P}_{h}^{0} u_{h}\right\|_{L^{2}\left((0, T), L^{2}(\Omega)\right)} \leq h\left\|u_{h}\right\|_{L^{2}\left((0, T), \mathcal{H}_{h}^{1}\left(\Omega_{h}\right)\right)} \stackrel{h \rightarrow 0}{\longrightarrow} 0
$$

we obtain the existence of a function $u \in W^{1, \infty}\left((0, T), L^{2}(\Omega)\right)$ such that, up to subsequences,

$$
\begin{cases}\mathbf{P}_{h}^{1} u_{h} \rightarrow u & \text { in } H^{1}\left((0, T), L^{2}(\Omega)\right), \\ \mathbf{P}_{h}^{0} u_{h}^{\prime} \rightarrow u^{\prime} & \text { in } L^{2}\left((0, T), L^{2}(\Omega)\right) .\end{cases}
$$

Also, by (6.3), $\left\{\mathbf{P}_{h}^{1} u_{h}\right\}_{h}$ is uniformly bounded in $C\left([0, T], H_{0}^{1}(\Omega)\right)$. Using the classical Aubin-Lions compactness result (see for instance [30]) we deduce that $\left\{\mathbf{P}_{h}^{1} u_{h}\right\}_{h}$ is relatively compact in $C\left([0, T], L^{2}(\Omega)\right)$. Thus we obtain

$$
\mathbf{P}_{h}^{1} u_{h} \rightarrow u \quad \text { in } H^{1}\left((0, T), L^{2}(\Omega)\right) \cap L^{2}\left((0, T), H_{0}^{1}(\Omega)\right),
$$

and

$$
\mathbf{P}_{h}^{1} u_{h} \rightarrow u \quad \text { in } C\left([0, T], L^{2}(\Omega)\right) .
$$

Also we prove that

$$
\left(\mathbf{P}_{h}^{0} u_{h}^{\prime \prime}\right) \text { is uniformly bounded in } L^{2}\left((0, T), H^{-1}(\Omega) .\right.
$$


For any $\varphi \in L^{2}\left((0, T), H_{0}^{1}(\Omega)\right)$ and $t \in(0, T)$ we have

$$
\begin{aligned}
\left\langle\mathbf{P}_{h}^{0} u_{h}^{\prime \prime}(t), \varphi\right\rangle_{-1,1} & =\int_{\Omega} \mathbf{P}_{h}^{0} u_{h}^{\prime \prime}(t) \varphi=\sum_{j, k=1}^{N} \int_{j h-h / 2}^{j h+h / 2} \int_{k h-h / 2}^{k h+h / 2}\left(\Delta_{h} u_{h}\right)_{j k}(t) \varphi \\
& =\sum_{j, k=1}^{N}\left(\Delta_{h} u_{h}\right)_{j k}(t) \int_{j h-h / 2}^{j h+h / 2} \int_{k h-h / 2}^{k h+h / 2} \varphi=: \sum_{j, k=1}^{N}\left(\Delta_{h} u_{h}\right)_{j k}(t) \widetilde{\varphi}_{j k}^{h} \\
& =-h^{2} \sum_{j, k=0}^{N}\left(\nabla_{h}^{+} u_{h}\right)_{j k}(t)\left(\nabla_{h}^{+} \widetilde{\varphi}\right)_{j k}^{h}=\int_{\Omega} \nabla\left(\mathbf{P}_{h}^{1} u_{h}\right)(t) \nabla\left(\mathbf{P}_{h}^{1} \widetilde{\varphi}^{h}\right) \\
& \lesssim\left\|\mathbf{P}_{h}^{1} u_{h}(t)\right\|_{H_{0}^{1}(\Omega)}\|\varphi\|_{H_{0}^{1}(\Omega)} .
\end{aligned}
$$

Thus we obtain 6.10). Using 6.10), 6.7] and the compactness result mentioned above we deduce that

$$
\mathbf{P}_{h}^{0} u_{h}^{\prime} \rightarrow u^{\prime} \quad \text { in } C\left([0, T], L^{2}(\Omega)\right) .
$$

Observe that, according to the bounds 6.6 , the subsequences may be extracted so that

$$
\mathbf{P}_{h}^{1} u_{h}(0) \rightarrow u^{0} \quad \text { in } H_{0}^{1}(\Omega) \quad \text { and } \quad \mathbf{P}_{h}^{0} u_{h}^{\prime}(0) \rightarrow u^{1} \quad \text { in } L^{2}(\Omega)
$$

for some $\left(u_{0}, u_{1}\right) \in H_{0}^{1}(\Omega) \times L^{2}(\Omega)$. Note that, in view of 6.9) and 6.11, $u(0)=u^{0}$ and $u^{\prime}(0)=u^{1}$.

Step II. Equation solved by the limit. We prove that $u$ solves the wave equation (1.3) with initial data $\left(u_{0}, u_{1}\right)$.

Let us choose $w \in C^{2}\left([0, T], C_{c}^{3}(\Omega)\right)$. Using the identity

$$
\int_{0}^{T} \int_{\Omega_{h}}\left(\Delta_{h} u_{h}\right) w d \Omega_{h} d t=-\int_{0}^{T} h^{2} \sum_{j, k=0}^{N} \nabla_{h}^{+} u_{h} \cdot \nabla_{h}^{+} w d t,
$$

integrating $\left[1.5\right.$ on $[0, T]$ and summing on $\Omega_{h}$ we get

$$
\int_{0}^{T} h^{2} \sum_{\mathbf{j} h \in \Omega_{h}}\left(u_{h}\right)_{\mathbf{j}} w_{\mathbf{j}}^{\prime \prime} d t+\int_{0}^{T} h^{2} \sum_{j, k=0}^{N} \nabla_{h}^{+} u_{h} \cdot \nabla_{h}^{+} w d t=\left.\left\langle\left(u_{h}, u_{h}^{\prime}\right),\left(w, w^{\prime}\right)\right\rangle\right|_{0} ^{T} .
$$

Thus

$$
\int_{0}^{T} \int_{\Omega} \mathbf{P}_{h}^{0} u_{h} \mathbf{P}_{h}^{0} w^{\prime \prime}+\int_{0}^{T} \int_{\Omega} \nabla\left(\mathbf{P}_{h}^{1} u_{h}\right) \cdot \nabla\left(\mathbf{P}_{h}^{1} w\right)=\left.\left\langle\left(\mathbf{P}_{h}^{0} u_{h}, \mathbf{P}_{h}^{0} u_{h}^{\prime}\right),\left(\mathbf{P}_{h}^{0} w, \mathbf{P}_{h}^{0} w^{\prime}\right)\right\rangle\right|_{0} ^{T} .
$$

Since

$$
\begin{cases}\mathbf{P}_{h}^{0} w^{\prime \prime} \rightarrow w^{\prime \prime} & \text { in } L^{2}\left((0, T), L^{2}(\Omega)\right), \\ \nabla\left(\mathbf{P}_{h}^{1} w\right) \rightarrow \nabla w & \text { in } L^{2}\left((0, T), L^{2}\left(\Omega^{2}\right)\right), \\ \left(\mathbf{P}_{h}^{0} w, \mathbf{P}_{h}^{0} w^{\prime}\right)(0) \rightarrow\left(w(0), w^{\prime}(0)\right) & \text { in } L^{2}(\Omega) \times L^{2}(\Omega), \\ \left(\mathbf{P}_{h}^{0} w, \mathbf{P}_{h}^{0} w^{\prime}\right)(T) \rightarrow\left(w(T), w^{\prime}(T)\right) & \text { in } L^{2}(\Omega) \times L^{2}(\Omega)\end{cases}
$$


and

$$
\begin{cases}\mathbf{P}_{h}^{0} u_{h} \rightarrow u & \text { in } L^{2}\left((0, T), L^{2}(\Omega)\right), \\ \nabla\left(\mathbf{P}_{h}^{1} u_{h}\right) \rightarrow \nabla u & \text { in } L^{2}\left((0, T), L^{2}\left(\Omega^{2}\right)\right), \\ \left(\mathbf{P}_{h}^{0} u_{h}, \mathbf{P}_{h}^{0} u_{h}^{\prime}\right)(0) \rightarrow\left(u(0), u^{\prime}(0)\right) & \text { in } L^{2}(\Omega) \times L^{2}(\Omega), \\ \left(\mathbf{P}_{h}^{0} u_{h}, \mathbf{P}_{h}^{0} u_{h}^{\prime}\right)(T) \rightarrow\left(u(T), u^{\prime}(T)\right) & \text { in } L^{2}(\Omega) \times L^{2}(\Omega),\end{cases}
$$

we find that the limit $u$ satisfies

$$
\int_{0}^{T} \int_{\Omega} u w^{\prime \prime}+\int_{0}^{T} \int_{\Omega} \nabla u \cdot \nabla w=\left.\left\langle\left(u, u^{\prime}\right),\left(w, w^{\prime}\right)\right\rangle\right|_{0} ^{T}
$$

for any $w \in C^{2}\left([0, T], H_{0}^{1}(\Omega)\right)$. This shows that $u$ is a solution of the homogeneous wave equation on $\Omega$.

Under the assumption of strong convergence of the initial data $\left(u_{h}^{0}, u_{h}^{1}\right)$, this together with the conservation of the energy gives

$$
\int_{0}^{T}\left[\left\|\mathbf{P}_{h}^{1} u_{h}(t)\right\|_{H_{0}^{1}(\Omega)}^{2}+\left\|\mathbf{P}_{h}^{0} u_{h}^{\prime}(t)\right\|_{L^{2}(\Omega)}^{2}\right] d t \rightarrow \int_{0}^{T}\left[\|u(t)\|_{H_{0}^{1}(\Omega)}^{2}+\left\|u^{\prime}(t)\right\|_{L^{2}(\Omega)}^{2}\right] d t
$$

Thus all the above weak convergences hold in the strong topology as well.

\subsection{Convergence of the normal derivatives}

In this subsection we prove that the interpolated discrete normal derivatives $\mathbf{P}_{0, \Gamma}^{h}\left(\partial_{n}^{h} u_{h}\right)$ converge to the continuous one $\partial_{n} u$, where $\mathbf{P}_{0, \Gamma}^{h}$ is the piecewise constant interpolator on the boundary $\Gamma_{h}$.

Proposition 6.2. Let $\left\{u_{h}(t)\right\}_{h}$ be a family of solutions of (5.3) satisfying 6.3. Let $u$ be any solution of (1.6) obtained by letting $h \rightarrow 0$ as in the statement of Proposition 6.1 . Then

$$
\mathbf{P}_{0, \Gamma}^{h}\left(\partial_{n}^{h} u_{h}\right) \rightarrow \partial_{n} u \quad \text { weakly in } L^{2}((0, T) \times \Gamma) .
$$

Moreover, if the family $\left\{u_{h}\right\}_{h>0}$ is such that $\mathbf{P}_{h}^{1} u_{h}(0) \rightarrow u^{0}$ in $H_{0}^{1}(\Omega)$ and $\mathbf{P}_{h}^{0} u_{h}^{\prime}(0) \rightarrow u^{1}$ in $L^{2}(\Omega)$ for some $\left(u^{0}, u^{1}\right) \in H^{1}(\Omega) \times L^{2}(\Omega)$ then the above convergences hold in the strong topologies. 
Proof. For any $u \in \mathcal{G}^{h}$ such that $\left.u\right|_{\Gamma_{h}}=0$ and $w \in \mathcal{G}^{h}$, explicit computations give

$$
\begin{aligned}
\int_{\Omega_{h}}\left(\Delta_{h} u\right) w d \Omega_{h} & +h^{2} \sum_{j, k=0}^{N}\left(\nabla_{h}^{+} u\right)_{j k}\left(\nabla_{h}^{+} w\right)_{j k} \\
& =h^{2} \sum_{j, k=1}^{N}\left(\Delta_{h} u\right)_{j k} w_{j k}+h^{2} \sum_{j, k=0}^{N}\left(\nabla_{h}^{+} u\right)_{j k}\left(\nabla_{h}^{+} w\right)_{j k} \\
& =-\sum_{k=1}^{N}\left(u_{N, k} w_{N+1, k}+y_{1, k} w_{0, k}\right)-\sum_{j=1}^{N}\left(u_{j, N} w_{j, N+1}+u_{j, 1} w_{j, 0}\right) \\
& =\int_{\Gamma_{h}}\left(\partial_{n}^{h} u\right) w d \Gamma_{h} .
\end{aligned}
$$

Let us choose $w \in C^{2}([0, T] \times \Omega)$. Applying identity 6.15 to the solution $u_{h}$ of equation (5.3) and $\left.w\right|_{G_{h}}$ we find that

$$
\begin{aligned}
\int_{0}^{T} \int_{\Omega_{h}} u_{h} w^{\prime \prime} d \Omega_{h} d t+\int_{0}^{T} h^{2} \sum_{j, k=0}^{N}\left(\nabla_{h}^{+} u_{h}\right)_{j k}\left(\nabla_{h}^{+} w\right)_{j k} d t \\
=\left.\left\langle\left(u_{h}, u_{h}^{\prime}\right),\left(w, w^{\prime}\right)\right\rangle\right|_{0} ^{T}+\int_{0}^{T} \int_{\Gamma_{h}}\left(\partial_{h}^{h} u_{h}\right) w d \Gamma_{h} d t
\end{aligned}
$$

Rewriting the above identity in terms of the interpolators $\mathbf{P}_{h}^{0}$ and $\mathbf{P}_{h}^{1}$ we get

$$
\begin{aligned}
\int_{0}^{T} \int_{\Omega} \mathbf{P}_{h}^{0} u_{h} \mathbf{P}_{h}^{0} w^{\prime \prime}+\int_{0}^{T} \int_{\Omega} \nabla\left(\mathbf{P}_{h}^{1} u_{h}\right) \cdot \nabla\left(\mathbf{P}_{h}^{1} w\right) \\
=\left.\left\langle\left(\mathbf{P}_{h}^{0} u_{h}, \mathbf{P}_{h}^{0} u_{h}^{\prime}\right),\left(\mathbf{P}_{h}^{0} w, \mathbf{P}_{h}^{0} w^{\prime}\right)\right\rangle\right|_{0} ^{T}+\int_{0}^{T} \int_{\Gamma} \mathbf{P}_{0, \Gamma}^{h}\left(\partial_{n}^{h} u_{h}\right) \mathbf{P}_{0, \Gamma}^{h} w d \Gamma d t .
\end{aligned}
$$

Since a solution $u$ of problem 1.3 satisfies

$$
\int_{0}^{T} \int_{\Omega}\left(u w^{\prime \prime}+\nabla u \cdot \nabla w\right) d x d t=\left.\left\langle\left(u, u^{\prime}\right),\left(w, w^{\prime}\right)\right\rangle\right|_{0} ^{T}+\int_{0}^{T} \int_{\Gamma} \partial_{n} u w d \Gamma d t
$$

for all $w \in L^{2}\left((0, T), H^{1}(\Omega)\right)$ with $w^{\prime \prime} \in L^{2}\left((0, T), L^{2}(\Omega)\right)$, and using the convergences for $\mathbf{P}_{h}^{0} u_{h}, \mathbf{P}_{h}^{1} u_{h}$ and $\mathbf{P}_{h}^{0} u_{h}^{\prime}$ given by Proposition 6.1, we obtain

$$
\int_{0}^{T} \int_{\Gamma} \mathbf{P}_{0, \Gamma}^{h}\left(\partial_{n}^{h} u_{h}\right) w d \Gamma d t \rightarrow \int_{0}^{T} \int_{\Gamma} \partial_{n} u w d \Gamma d t
$$

This shows that

$$
\mathbf{P}_{0, \Gamma}^{h}\left(\partial_{n}^{h} u_{h}\right) \rightarrow \partial_{n} u \quad \text { weakly in } L^{2}((0, T) \times \Gamma) .
$$

The proof of the strong convergence is more subtle. For any $\epsilon>0$ we can choose $\left.\left(\tilde{u}^{0}, \tilde{u}^{1}\right) \in H^{2}(\Omega) \times H^{1}(\Omega)\right)$ such that $\left\|\tilde{u}^{0}-u^{0}\right\|_{H^{1}(\Omega)} \leq \epsilon$ and $\left\|\tilde{u}^{1}-u^{1}\right\|_{L^{2}(\Omega)} \leq \epsilon$. 
We denote by $\left(\tilde{u}_{h}^{0}, \tilde{u}_{h}^{1}\right)$ the approximations of $\left(\tilde{u}^{0}, \tilde{u}^{1}\right)$. In this case the discrete solutions $\left(\tilde{u}_{h}, \tilde{u}_{h}^{\prime}\right)$ of equation (5.3) are smooth enough to guarantee that $\mathbf{P}_{0, \Gamma}^{h}\left(\partial_{n}^{h} \tilde{u}_{h}\right)$ is compact in $L^{2}((0, T) \times \Gamma)$, and thus

$$
\mathbf{P}_{0, \Gamma}^{h}\left(\partial_{n}^{h} \tilde{u}_{h}\right) \rightarrow \partial_{n} \tilde{u} \quad \text { in } L^{2}((0, T) \times \Gamma) .
$$

Setting $\tilde{\tilde{u}}=u-\tilde{u}, \tilde{\tilde{u}}_{h}=u_{h}-\tilde{u}_{h}$ and using the fact that the energy on the boundary is controlled by the total energy both in the discrete and continuous setting we have

$$
\left\|\mathbf{P}_{0, \Gamma}\left(\partial_{n}^{h} \tilde{\tilde{u}}_{h}\right)\right\|_{L^{2}((0, T) \times \Gamma)} \leq C(T) \mathcal{E}\left(\tilde{\tilde{u}}_{h}\right) \leq C(T) \epsilon
$$

and

$$
\left\|\partial_{n} \tilde{\tilde{u}}\right\|_{L^{2}((0, T) \times \Gamma)} \leq C(T) \mathcal{E}(\tilde{\tilde{u}}) \leq C(T) \epsilon .
$$

Using now 6.17, 6.18) and 6.19) we obtain the strong convergence of $\mathbf{P}_{0, \Gamma}\left(\partial_{n}^{h} u_{h}\right)$ towards $\partial_{n} u$ in $L^{2}((0, T) \times \Gamma)$.

\section{Convergence of the controlled problem}

Concerning the convergence of the semidiscrete control of $[1.5)$ we prove the following result.

Theorem 7.1. Let $\left(y^{0}, y^{1}\right) \in L^{2}(\Omega) \times H^{-1}(\Omega)$ and $\left(y_{h}^{0}, y_{h}^{1}\right)$ be such that

$$
\mathbf{P}_{h}^{0} y_{h}^{0} \rightarrow y^{0} \quad \text { in } L^{2}(\Omega), \quad \mathbf{P}_{h}^{-1} y_{h}^{1} \rightarrow y^{1} \quad \text { in } H^{-1}(\Omega) .
$$

Then for any $T>4$ the solution $\left(y_{h}, y_{h}^{\prime}\right)$ and its partial controls $v_{h}$ given by Theorem 5.1 satisfy

$$
\mathbf{P}_{h}^{0} y_{h} \stackrel{*}{\rightarrow} y \quad \text { in } L^{\infty}\left([0, T], L^{2}(\Omega)\right), \quad\left(\mathbf{P}_{h}^{0} y_{h}\right)^{\prime} \stackrel{*}{\rightarrow} y^{\prime} \quad \text { in } L^{\infty}\left([0, T], H^{-1}(\Omega)\right)
$$

and

$$
\mathbf{P}_{0, \Gamma}^{h} v_{h} \rightarrow v \quad \text { in } L^{2}\left([0, T], L^{2}\left(\Gamma_{0}\right)\right),
$$

where $\left(y, y_{t}\right)$ solves $(1.1]$, with the limit control $v$, and satisfies $[1.2)$. The limit control $v$ is given by

$$
v=\partial_{n} u^{*} \quad \text { on } \Gamma_{0},
$$

where $u^{*}$ solves the adjoint problem

$$
\begin{cases}u^{\prime \prime}-\Delta u=0 & \text { in } \Omega \times(0, T), \\ u=0 & \text { on } \Gamma \times(0, T), \\ u(T, x)=u^{0}(x), u_{t}(T, x)=u^{1}(x) & \text { in } \Omega,\end{cases}
$$

with data $\left(u^{0, *}, u^{1, *}\right) \in H_{0}^{1}(\Omega) \times L^{2}(\Omega)$ minimizing the functional

$$
J\left(\left(u^{0}, u^{1}\right)\right)=\frac{1}{2} \int_{0}^{T} \int_{\Gamma_{0}}\left|\partial_{n} u\right|^{2} d t+\left\langle\left(y^{0}, y^{1}\right),\left(u^{0}, u^{1}\right)\right\rangle
$$

in $H_{0}^{1}(\Omega) \times L^{2}(\Omega)$. 
Proof. Step I. Weak convergence of $v_{h}$. Theorem 5.1 gives us the function $v_{h}=$ $\partial_{n}^{h} u_{h}^{*}(t)$, which depends on $\left(y_{h}^{0}, y_{h}^{1}\right)$ and satisfies (5.1). Recall that $u_{h}^{*}$ solves 5.3) with final state $\left(u_{h}^{0, *}, u_{h}^{1, *}\right) \in V^{h} \times V^{h}$ minimizing the function $J_{h}$.

Moreover, as a consequence of the observability inequality (3.6), we have

$\left\|u_{h}^{0, *}\right\|_{1, h}+\left\|u_{h}^{1, *}\right\|_{0, h} \leq C(T)\left\|\partial_{h}^{h} u_{h}^{*}\right\|_{L^{2}\left((0, T) \times \Gamma_{0 h}\right)} \leq C(T)\left(\left\|y_{h}^{1}\right\|_{0, h}+\left\|y_{h}^{0}\right\|_{-1, h}\right) \leq C(T)$.

In these conditions, Proposition 6.1 guarantees the existence of a function $u^{*}$ that solves 1.3) and, in addition,

$$
\mathbf{P}_{0, \Gamma}^{h} v_{h}(t)=\mathbf{P}_{0, \Gamma}^{h}\left(\partial_{n}^{h} u_{h}^{*}\right) \rightarrow \partial_{n} u^{*} \quad \text { weakly in } L^{2}\left((0, T) \times \Gamma_{0}\right) \text { as } h \rightarrow 0 .
$$

Step II. Weak convergence of $y_{h}$. Let us now consider equation $(1.5)$ with initial data $\left(y_{h}^{0}, y_{h}^{1}\right)$ and $v_{h}$ as above. Then for any solution $u_{h}$ of the adjoint problem 5.3), the following holds for all $0<s<T$ :

$$
\int_{0}^{s} \int_{\Gamma_{0 h}} v_{h}(t) \partial_{h}^{h} u_{h}(t) d \Gamma_{0 h} d t+\left.\left\langle\left(y_{h}, y_{h}^{\prime}\right),\left(u_{h}, u_{h}^{\prime}\right)\right\rangle_{h}\right|_{0} ^{s}=0 .
$$

Thus, in view of the direct inequality 2.10 and the conservation of energy applied to $u_{h}$, we get, for any $s<T$,

$$
\begin{aligned}
\left|\left\langle\left(y_{h}(s), y_{h}^{\prime}(s)\right),\left(u_{h}^{0}, u_{h}^{1}\right)\right\rangle_{h}\right| \leq & \left|\left\langle\left(y_{h}^{0}, y_{h}^{1}\right),\left(u_{h}(0), u_{h}^{\prime}(0)\right)\right\rangle_{h}\right| \\
& +\left\|v_{h}\right\|_{L^{2}\left((0, T) \times \Gamma_{0 h}\right)}\left\|\partial_{h}^{h} u_{h}\right\|_{L^{2}\left((0, T) \times \Gamma_{0 h}\right)} \\
\leq & C(T)\left(\left\|y_{h}^{0}\right\|_{0, h}+\left\|y_{h}^{1}\right\|_{-1, h}\right)\left(\left\|u_{h}^{0}\right\|_{1, h}+\left\|u_{h}^{1}\right\|_{0, h}\right) .
\end{aligned}
$$

This means that for any $0 \leq s \leq T$,

$$
\left\|y_{h}(s)\right\|_{0, h}+\left\|y_{h}^{\prime}(s)\right\|_{-1, h} \leq C .
$$

Using this estimate we claim the existence of a positive constant such that

$$
\left\{\begin{array}{l}
\left\|\mathbf{P}_{h}^{0} y_{h}\right\|_{L^{\infty}\left([0, T], L^{2}(\Omega)\right)} \leq C, \\
\left\|\mathbf{P}_{h}^{0} y_{h}^{\prime}\right\|_{L^{\infty}\left([0, T], H^{-1}(\Omega)\right)} \leq C
\end{array}\right.
$$

and

$$
\left\{\begin{array}{l}
\left\|\mathbf{P}_{h}^{-1} y_{h}\right\|_{L^{\infty}\left([0, T], L^{2}(\Omega)\right)} \leq C, \\
\left\|\mathbf{P}_{h}^{-1} y_{h}^{\prime}\right\|_{L^{\infty}\left([0, T], H^{-1}(\Omega)\right)} \leq C, \\
\left\|\mathbf{P}_{h}^{-1} y_{h}^{\prime \prime}\right\|_{L^{2}\left([0, T], H^{-2}(\Omega)\right)} \leq C .
\end{array}\right.
$$

The first four properties follow from the definition of the interpolators and property 7.5. The last estimate follows since $y_{h}$ solves the discrete wave equation:

$$
\begin{aligned}
\left\|\mathbf{P}_{h}^{-1} y_{h}^{\prime \prime}\right\|_{L^{\infty}\left([0, T], H^{-2}(\Omega)\right)} & :=\left\|(-\Delta) \mathbf{P}_{h}^{1}\left(-\Delta_{h}\right)^{-1} y_{h}^{\prime \prime}\right\|_{L^{2}\left([0, T], H^{-2}(\Omega)\right)} \\
& \leq\left\|\left(-\Delta_{h}\right)^{-1} y_{h}^{\prime \prime}\right\|_{L^{2}\left([0, T], L^{2}(\Omega)\right)} \\
& \leq\left\|y_{h}\right\|_{L^{2}\left([0, T], L^{2}(\Omega)\right)}+\left\|y_{h}\right\|_{L^{2}\left([0, T], L^{2}\left(\Gamma_{h}\right)\right)} \\
& \leq C+\left\|v_{h}\right\|_{L^{2}\left([0, T], L^{2}\left(\Gamma_{0 h}\right)\right)} \leq 2 C .
\end{aligned}
$$


Lemma 6.1 implies that

$$
\left\|\mathbf{P}_{h}^{0} y_{h}-\mathbf{P}_{h}^{-1} y_{h}\right\|_{L^{2}\left([0, T], H^{-1}(\Omega)\right)} \leq h T\left\|y_{h}\right\|_{0, h} \rightarrow 0
$$

as $h \rightarrow 0$. Using estimates (7.6) and 7.7 we obtain the existence of a function $y \in$ $W^{1, \infty}\left((0, T), H^{-1}(\Omega)\right)$ such that, up to a subsequence,

$$
\begin{cases}\mathbf{P}_{h}^{0} y_{h} \rightarrow y & \text { in } \left.H^{1}(0, T), H^{-1}(\Omega)\right) \\ \mathbf{P}_{h}^{-1} y_{h}^{\prime} \rightarrow y^{\prime} & \text { in } \left.L^{2}(0, T), H^{-1}(\Omega)\right)\end{cases}
$$

Estimates (7.6) show that (see [30, Corollary 1]), up to a subsequence, $\mathbf{P}_{h}^{0} y_{h} \rightarrow y$ in $C\left([0, T], H^{-1}(\Omega)\right)$. In particular, $\mathbf{P}_{h}^{0} y_{h}(0) \rightarrow y(0)$ in $H^{-1}(\Omega)$. As $\mathbf{P}_{h}^{0} y_{h}(0)$ is uniformly bounded in $L^{2}(\Omega)$ we get $\mathbf{P}_{h}^{0} y_{h}(0) \rightarrow y(0)$ in $L^{2}(\Omega)$ and, by [7.1), we obtain $y(0)=y^{0}$.

The last two estimates of (7.7) show that (see [30, Corollary 1]), up to a subsequence, $\mathbf{P}_{h}^{-1} y_{h}^{\prime} \rightarrow y^{\prime}$ strongly in $C\left([0, T], H^{-2}(\Omega)\right)$. In particular, $\mathbf{P}_{h}^{-1} y_{h}^{\prime}(0) \rightarrow y^{\prime}(0)$ in $H^{-2}(\Omega)$ ). Since $\mathbf{P}_{h}^{-1} y_{h}^{\prime}(0)$ is uniformly bounded in $H^{-1}(\Omega)$ we get $\mathbf{P}_{h}^{-1} y_{h}^{\prime}(0) \rightarrow$ $y^{\prime}(0)$ in $H^{-1}(\Omega)$ and, by $\left(7.1\right.$, we obtain $y^{\prime}(0)=y^{1}$.

Let us choose $\left(u^{0}, u^{1}\right) \in H_{0}^{1}(\Omega) \times L^{2}(\Omega)$ as final state in the adjoint equation (7.2). We choose $\left(u_{h}^{0}, u_{h}^{1}\right)$ in the adjoint discrete system 5.3 such that $\mathbf{P}_{h}^{1} u_{h}^{0} \rightarrow u^{0}$ in $H_{0}^{1}(\Omega)$ and $\mathbf{P}_{h}^{0} u_{h}^{1} \rightarrow u^{1}$ in $L^{2}(\Omega)$. In view of Proposition 6.1 we have the following strong convergence properties:

$$
\begin{cases}\mathbf{P}_{h}^{1} u_{h} \rightarrow u & \text { in } L^{2}\left([0, T], H_{0}^{1}(\Omega)\right), \\ \mathbf{P}_{h}^{0} u_{h}^{\prime} \rightarrow u^{\prime} & \text { in } L^{2}\left([0, T], L^{2}(\Omega)\right),\end{cases}
$$

where $u$ is the solution of equation 1.3 with final state $\left(u^{0}, u^{1}\right)$.

We write (7.4) as

$$
\int_{0}^{s} \int_{\Gamma_{0}} \mathbf{P}_{0, \Gamma}^{h} v_{h} \mathbf{P}_{0, \Gamma}^{h}\left(\partial_{n}^{h} u_{h}\right) d \sigma d t+\left.\left\langle\left(\mathbf{P}_{h}^{0} y_{h}, \mathbf{P}_{h}^{-1} y_{h}^{\prime}\right),\left(\mathbf{P}_{h}^{1} u_{h}, \mathbf{P}_{h}^{0} u_{h}^{\prime}\right)\right\rangle\right|_{0} ^{s}=0 .
$$

Since $\mathbf{P}_{h}^{0} y_{h} \rightarrow y$ weakly in $L^{2}\left((0, T), L^{2}(\Omega)\right)$ and also $\mathbf{P}_{h}^{-1} y_{h}^{\prime} \rightarrow y^{\prime}$ weakly in $L^{2}\left((0, T), H^{-1}(\Omega)\right)$, letting $h \rightarrow 0$ we obtain

$$
\int_{0}^{s} \int_{\Gamma_{0}} \partial_{n} u^{*} \partial_{n} u d \sigma d t+\left.\left\langle\left(y, y^{\prime}\right),\left(u, u^{\prime}\right)\right\rangle_{h}\right|_{0} ^{s}=0, \quad \forall s<T
$$

where $u$ is a solution of problem (7.2) with final state $\left(u^{0}, u^{1}\right)$. Thus $y$ is a solution by transposition of 1.1 with control $v=\partial_{n} u^{*}$. 
Step III. Final time control requirement. We prove that 1.2 holds. We consider the case of $y(T)$, the other case being similar. Since $\left(y_{h}(T), w_{h}\right)_{h}=0$ for all functions $w_{h} \in V^{h}$ we obtain

$$
\int_{\Omega} \mathbf{P}_{h}^{0} y_{h}(T) \mathbf{P}_{h}^{0} w_{h} d x=0 \quad \text { for all } h>0
$$

As $\mathbf{P}_{h}^{0} y_{h}(T) \rightarrow y(T)$ strongly in $L^{2}(\Omega)$, and $\mathbf{P}_{h}^{0}\left(V^{h}\right)$ is dense in $L^{2}(\Omega)$, we get

$$
\int_{\Omega} y(T) w d x=0
$$

for all $w \in L^{2}(\Omega)$. Thus $y(T) \equiv 0$.

Finally, using the uniqueness results for problem 11.1 we conclude that the control $v$ obtained before satisfies $v=\partial_{n} u^{*}$ where $u^{*}$ is the solution of problem (7.2) with final state $\left(u^{*, 0}, u^{*, 1}\right)$ minimizing the functional $(7.3)$.

\section{Concluding remarks}

In this article we have developed a quite systematic approach to proving the convergence of the controls obtained by two-grid methods. It relies essentially on the following ingredients:

- a convergent numerical scheme;

- the Fourier decomposition of solutions;

- the conservative nature of the model and of the numerical approximation schemes under consideration;

- the uniform (with respect to the mesh-size) observability of low frequency solutions.

In these circumstances, the dyadic decomposition argument can be applied to yield the uniform observability of the two-grid solutions.

Accordingly, the method we employ can be adapted to the following situations:

- Other models. The observability of filtered low frequency solutions of numerical approximation schemes has been proved not only for the wave equation ([14], [31]), but also for other models: Schrödinger equations [21] and beam equations [17], for instance. In these two cases the main ingredients we have indicated above clearly arise and therefore the method we have developed can be easily adapted, thus yielding the convergence of the two-grid method in the square domain.

- Other control mechanisms. This article has been devoted to the problem of boundary observability. But the method we have developed applies with minor changes to the problem of internal observability for which the measurement on solutions is done in an open subset $\omega$ of the domain. This can in particular be done when the control is localized in a neighborhood of the subset of the boundary containing two adjacent sides of the boundary.

- Control of nonlinear wave equations. In the case of nonlinear problems in dimension one, in [33] the convergence of the two-grid algorithm was proved for semilinear wave 
equations with globally Lipschitz nonlinearities. The combination of the methods of this paper and [33] yields the same result in the multidimensional case.

- Fully discrete schemes. In this article we have analyzed semidiscrete models but the same analysis of uniform observability can be performed for conservative fully discrete discretizations of the wave equation or other models in the square domain. Indeed, once more, all the needed ingredients to apply the programme developed in this article are also present in that framework. For instance, in the one-dimensional case, the low frequency uniform observability of solutions of fully discrete approximations has been proved in [26], using discrete versions of Ingham's inequalities. Applying the techniques developed in this article with the results in [26] one can immediately deduce the uniform controllability of fully discrete schemes for the 1-d wave equation after the two-grid strategy is applied.

The methods of this article can also be combined with other tools to get more general results and address other related issues. But this has to be investigated in more detail. Possible extensions include:

- Control of nonlinear wave equations. As we said before, the method also works for nonlinear wave equations with Lipschitz nonlinearities. But whether these results can be extended to more general nonlinearities, growing at infinity in a superlinear way, is an open problem.

- Meshes with ratio $m / n$. The two-grid method we proposed here had a mesh-ratio of the form $1 / p$. One could expect the uniform observability to hold in 1-d for any meshratio $m / n<1$, in the multidimensional case, when $m / n<1 / 2$. The only difficulty in doing that is to prove a result similar to Lemma 3.1 for all functions in the image of $\Pi_{h}^{n / m h} \mathcal{G}_{h}$. As far as we know, these are open problems.

- Other boundary conditions. In the proof we use the so-called direct inequality whose analogue fails for other closely related problems, like the boundary control of the wave equation with Neumann boundary conditions.

- Spectral conditions for observability. In recent works [28], [23], [29], the authors give a spectral condition which guarantees the observability for infinite-dimensional conservative systems. This type of condition generalizes the Hautus test for finitedimensional systems to infinite-dimensional ones. It would be interesting to see if these spectral methods can be adapted in order to guarantee uniform observability results for numerical methods based on the two-grid method. The main difficulty in applying these results is due to the fact that the space $V^{h}$ of two-grid data is not invariant under the semidiscrete wave flow.

But the method presented here has its limitations as well. We now mention some of them:

- More general meshes. We used intensively Fourier analysis techniques, which are not available for irregular meshes, requiring further developments.

- Dissipative equations. As mentioned above, our analysis is mainly valid for conservative systems. We could also consider the wave equation with a bounded dissipative 
potential, but the methods we have developed here cannot address genuinely dissipative models like the heat equation, viscoelasticity, etc.

- Dissipative schemes. The same can be said about the numerical schemes we have considered. Our analysis applies to both semidiscrete and fully discrete conservative schemes, but not to dissipative ones.

\section{Appendix A. Proof of Lemma 4.1}

In this Appendix we prove Lemma 4.1. The main ingredient is the following lemma inspired by ideas of [15], [3] and adapted to our context. In the following, $X$ denotes the space $L^{p}(\Omega, d \mu)$, where $\mu$ is a Borel measure and $\mu(\Omega)<\infty$.

Lemma A.1. Let $c>1, T>0, P \in C_{c}^{\infty}(\mathbb{R})$ and $\left(P_{k}\right)_{k \geq 0}$ be as in 4.1. Also let $\varphi \in C_{0}^{\infty}(0, T)$ and let $\psi \in L^{\infty}(\mathbb{R})$ satisfy $\psi \equiv 1$ on $(0, T)$. There exists a positive constant $C=C(T, \varphi, \psi, P)$ such that

$$
\begin{aligned}
\int_{\mathbb{R}}\left\|\varphi(t) P_{k}(w)(t)\right\|_{X}^{2} d t \leq & 2 \int_{\mathbb{R}}\left\|\varphi(t) P_{k}(\psi w)(t)\right\|_{X}^{2} d t \\
& +C c^{-2 k} \sup _{l \in \mathbb{Z}}\|w\|_{L^{2}((l T,(l+1) T), X)}^{2}
\end{aligned}
$$

for all $w \in L_{\text {loc }}^{2}(\mathbb{R}, X)$ and $k \geq 0$.

Proof. We set $I_{l}=[l T,(l+1) T)$ and $w_{l}=1_{I_{l}} w$. We claim that there exists a positive constant $C(P)$ such that for all $\varphi \in C_{0}^{\infty}(\mathbb{R})$ and $l \in \mathbb{Z}$ with $\operatorname{dist}\left(I_{l}, \operatorname{supp}(\varphi)\right)>0$ the following holds:

$$
\sup _{t \in[0, T]}\left\|\varphi(t) P_{k}\left(w_{l}\right)\right\|_{X} \leq \frac{C(P) T^{1 / 2} c^{-k}}{\operatorname{dist}\left(I_{l}, \operatorname{supp}(\varphi)\right)^{2}}\|\varphi\|_{L^{\infty}(\mathbb{R})} \sup _{l \in \mathbb{Z}}\left\|w_{l}\right\|_{L^{2}(\mathbb{R}, X)},
$$

uniformly for all $k \geq 0$.

Using estimate A.2 we will prove the existence of a positive constant $C=$ $C(T, \varphi, \psi, P)$ such that

$$
\sup _{t \in[0, T]}\left\|\varphi(t)\left(P_{k}(w)-P_{k}(\psi w)\right)(t)\right\|_{X} \leq C c^{-k} \sup _{l \in \mathbb{Z}}\left\|w_{l}\right\|_{L^{2}(\mathbb{R}, X)} .
$$

Then (A.1) will be a consequence of Minkowski's and Cauchy's inequalities:

$$
\begin{aligned}
\int_{\mathbb{R}}\left\|\varphi(t) P_{k}(w)(t)\right\|_{X}^{2} d t \\
\quad \leq 2 \int_{\mathbb{R}}\left\|\varphi(t) P_{k}(\psi w)(t)\right\|_{X}^{2} d t+2 \int_{\mathbb{R}}\left\|\varphi(t) P_{k}(w-\psi w)(t)\right\|_{X}^{2} d t \\
\leq 2 \int_{\mathbb{R}}\left\|\varphi(t) P_{k}(\psi w)(t)\right\|_{X}^{2} d t+2 T \sup _{t \in[0, T]}\left\|\varphi(t)\left(P_{k}(w-\psi w)\right)(t)\right\|_{X}^{2} \\
\leq 2 \int_{\mathbb{R}}\left\|\varphi(t) P_{k}(\psi w)(t)\right\|_{X}^{2} d t+C c^{-k} \sup _{l \in \mathbb{Z}}\left\|w_{l}\right\|_{L^{2}(\mathbb{R}, X)}^{2} .
\end{aligned}
$$


Step I. Proof of $\mathbf{A . 2}$. The definition of the projector $P_{k}$ and integration by parts give us

$$
\begin{aligned}
\varphi(t) P_{k}\left(w_{l}\right)(t) & =\int_{\mathbb{R}_{\tau}} \int_{\mathbb{R}_{s}} e^{i \tau(t-s)} P\left(c^{-k} \tau\right) \varphi(t) w_{l}(s) d s d \tau \\
& =\int_{\mathbb{R}_{\tau}} \int_{\mathbb{R}_{s}} e^{i \tau(t-s)} i^{2} \partial_{\tau}^{2}\left[P\left(c^{-k} \tau\right)\right] \frac{\varphi(t) w_{l}(s)}{(t-s)^{2}} d s d \tau .
\end{aligned}
$$

Since $\operatorname{dist}\left(\operatorname{supp}(\varphi), I_{l}\right)>0$, for any $t$ in the support of $\varphi$ we apply Minkowski's inequality to obtain

$$
\begin{aligned}
\left\|\varphi(t) P_{k}\left(w_{l}\right)(t)\right\|_{X} & \leq c^{-2 k}\|\varphi\|_{L^{\infty}(\mathbb{R})} \int_{\mathbb{R}_{\tau}}\left|\left(\partial_{\tau}^{2} P\right)\left(c^{-k} \tau\right)\right| d \tau \int_{I_{l}} \frac{\left\|w_{l}(s)\right\|_{X}}{(t-s)^{2}} d s \\
& \leq \frac{c^{-k}\|\varphi\|_{L^{\infty}(\mathbb{R})}}{\left(\operatorname{dist}\left(\operatorname{supp}(\varphi), I_{l}\right)\right)^{2}} \int_{\mathbb{R}_{\tau}}\left|\left(\partial_{\tau}^{2} P\right)(\tau)\right| d \tau \int_{I_{l}}\left\|w_{l}(s)\right\|_{X} d s . \\
& \leq \frac{T^{1 / 2} c^{-k}\|\varphi\|_{L^{\infty}(\mathbb{R})}}{\left(\operatorname{dist}\left(\operatorname{supp}(\varphi), I_{l}\right)\right)^{2}} \int_{\mathbb{R}_{\tau}}\left|\left(\partial_{\tau}^{2} P\right)(\tau)\right| d \tau\left(\int_{I_{l}}\left\|w_{l}(s)\right\|_{X}^{2} d s\right)^{1 / 2} .
\end{aligned}
$$

Step II. Proof of A.3. Observe that $w \equiv w \psi$ on $I_{0}$. This yields the following decomposition of the difference $P_{k}(w)-P_{k}(\psi w)$ :

$$
P_{k}(w)-P_{k}(\psi w)=\sum_{|l| \geq 1} P_{k}\left(w_{l}-(\psi w)_{l}\right)=\sum_{|l| \geq 1} P_{k}\left(b_{l}\right)
$$

with $b_{l}=w_{l}-(\psi w)_{l}$. Let us choose $\delta>0$ such that $\varphi$ is supported on $(\delta, T-\delta)$. Thus for all $|l| \geq 2$, the function $b_{l}$ satisfies $\operatorname{dist}\left(\operatorname{supp}(\varphi), I_{l}\right) \geq T(|l|-1)$. Also, for $|l|=1$, $\operatorname{dist}\left(\operatorname{supp}(\varphi), I_{l}\right) \geq \delta$. By A.2 we obtain

$$
\begin{aligned}
& \sup _{t \in \mathbb{R}}\left\|\varphi(t) P_{k}\left(b_{l}\right)(t)\right\|_{X} \\
& \quad \leq C(P) T^{1 / 2} c^{-k}\|\varphi\|_{L^{\infty}(\mathbb{R})} \sup _{l \in \mathbb{Z}}\left\|b_{l}\right\|_{L^{2}(\mathbb{R}, X)} \begin{cases}\frac{1}{T^{2}(|l|-1)^{2}}, & |l| \geq 2, \\
\frac{1}{\delta^{2}}, & |l|=1 .\end{cases}
\end{aligned}
$$

By A.4 and A.5 there exists a constant $C=C(T, \varphi, \psi, P)$ such that for any $t \in[0, T]$,

$$
\begin{aligned}
\left\|\varphi(t)\left[P_{k}(w)-P_{k}(\psi w)\right]\right\|_{X} & \leq \sum_{|l| \geq 1}\left\|\varphi(t) P_{k}\left(b_{l}\right)\right\|_{X} \leq C c^{-k} \sup _{l \in \mathbb{Z}}\left\|b_{l}\right\|_{L^{2}(\mathbb{R}, X)} \\
& \leq C c^{-k} \sup _{l \in \mathbb{Z}}\|w\|_{L^{2}(\mathbb{R}, X)} .
\end{aligned}
$$

Proof of Lemma 4.1. Let us choose a function $\varphi \in C_{0}^{\infty}(0, T)$ such that $|\varphi| \leq 1$ and $\varphi \equiv 1$ on $[2 \delta, T-2 \delta]$. Applying Lemma A.1 to the function $w$ and $\psi=\mathbf{1}_{(0, T)}$, we obtain the 
existence of a positive constant $C(\delta, T, P)$ such that

$$
\begin{aligned}
\int_{2 \delta}^{T-2 \delta}\left\|P_{k} w\right\|_{X}^{2} d t & \leq \int_{\mathbb{R}} \varphi^{2}\left\|P_{k}(w)\right\|_{X}^{2} d t \\
& \leq 2 \int_{\mathbb{R}} \varphi^{2}\left\|P_{k}(\psi w)\right\|_{X}^{2} d t+\frac{C(\delta, T, P)}{c^{2 k}} \sup _{l \in \mathbb{Z}}\|w\|_{L^{2}((l T,(l+1) T), X)}^{2} .
\end{aligned}
$$

Summing all these inequalities we get

$$
\begin{aligned}
\sum_{k \geq k_{0}} \int_{2 \delta}^{T-2 \delta}\left\|P_{k} w\right\|_{X}^{2} d t \leq & 2 \sum_{k \geq k_{0}} \int_{\mathbb{R}} \varphi^{2}\left\|P_{k}(\psi w)\right\|_{X}^{2} d t \\
& +\frac{C(\delta, T, P)}{c^{2 k_{0}}} \sup _{l \in \mathbb{Z}}\|w\|_{L^{2}((l T,(l+1) T), X)}^{2}
\end{aligned}
$$

In the following we prove the existence of a positive constant $C(P, c)$ such that

$$
\sum_{k \geq 0} \int_{\mathbb{R}} \varphi^{2}\left\|P_{k}(\psi w)\right\|_{X}^{2} d t \leq C(P, c) \int_{0}^{T}\|w(t)\|_{X}^{2} d t .
$$

Observe that any real number $\tau$ belongs either to a finite number of intervals of the form $\left( \pm a c^{k}, \pm b c^{k}\right)$ or to none of them. Thus there is a positive constant $C(P, c)$ such that

$$
\sup _{\tau \in \mathbb{R}} \sum_{k \geq 0} P^{2}\left(c^{-k} \tau\right) \leq C(P, c) .
$$

Applying Plancherel's identity in the time variable we obtain

$$
\begin{aligned}
& \sum_{k \geq 0} \int_{\mathbb{R}} \varphi^{2}(t)\left\|P_{k}(\psi w)(t)\right\|_{X}^{2} d t \leq\|\varphi\|_{L^{\infty}(\mathbb{R})}^{2} \sum_{k \geq 0} \int_{\mathbb{R}}\left\|P_{k}(\psi w)(t)\right\|_{X}^{2} d t \\
& \quad=\|\varphi\|_{L^{\infty}(\mathbb{R})}^{2} \sum_{k \geq 0} \int_{\mathbb{R}} P^{2}\left(c^{-k} \tau\right)\|\widehat{\psi w}(\tau)\|_{X}^{2} d \tau \\
& \quad \leq\|\varphi\|_{L^{\infty}(\mathbb{R})}^{2} \sup _{\tau \in \mathbb{R}} \sum_{k \geq 0} P^{2}\left(c^{-k} \tau\right) \int_{\mathbb{R}}\|\widehat{\psi w}(\tau)\|_{X}^{2} d \tau \\
& \quad \leq C(P, c)\|\varphi\|_{L^{\infty}(\mathbb{R})}^{2} \int_{\mathbb{R}}\|(\psi w)(t)\|_{X}^{2} d t=C(P, c)\|\varphi\|_{L^{\infty}(\mathbb{R})}^{2} \int_{0}^{T}\|w(t)\|_{X}^{2} d t .
\end{aligned}
$$

\section{Appendix B. Spectral analysis of $V^{h}$-functions}

In this section we analyze the $\mathcal{H}_{h}^{s}\left(\Omega_{h}\right)$-norms of the functions belonging to $V^{h}$, i.e. the space of functions defined on the fine grid as a linear interpolation of the functions defined on the coarse one, and we prove Lemma 3.1. We will consider periodic discrete functions defined on the grid $x_{0}=0, x_{1}=h, \ldots, x_{2 N+1}=(2 N+1) h=2$ instead of vanishing at the boundary, but all the results also apply to this case.

We first obtain a description of the Fourier coefficients $\widehat{v}(\mathbf{j})$ of a periodic function $v \in V^{h}$ and then prove Lemma 3.1 

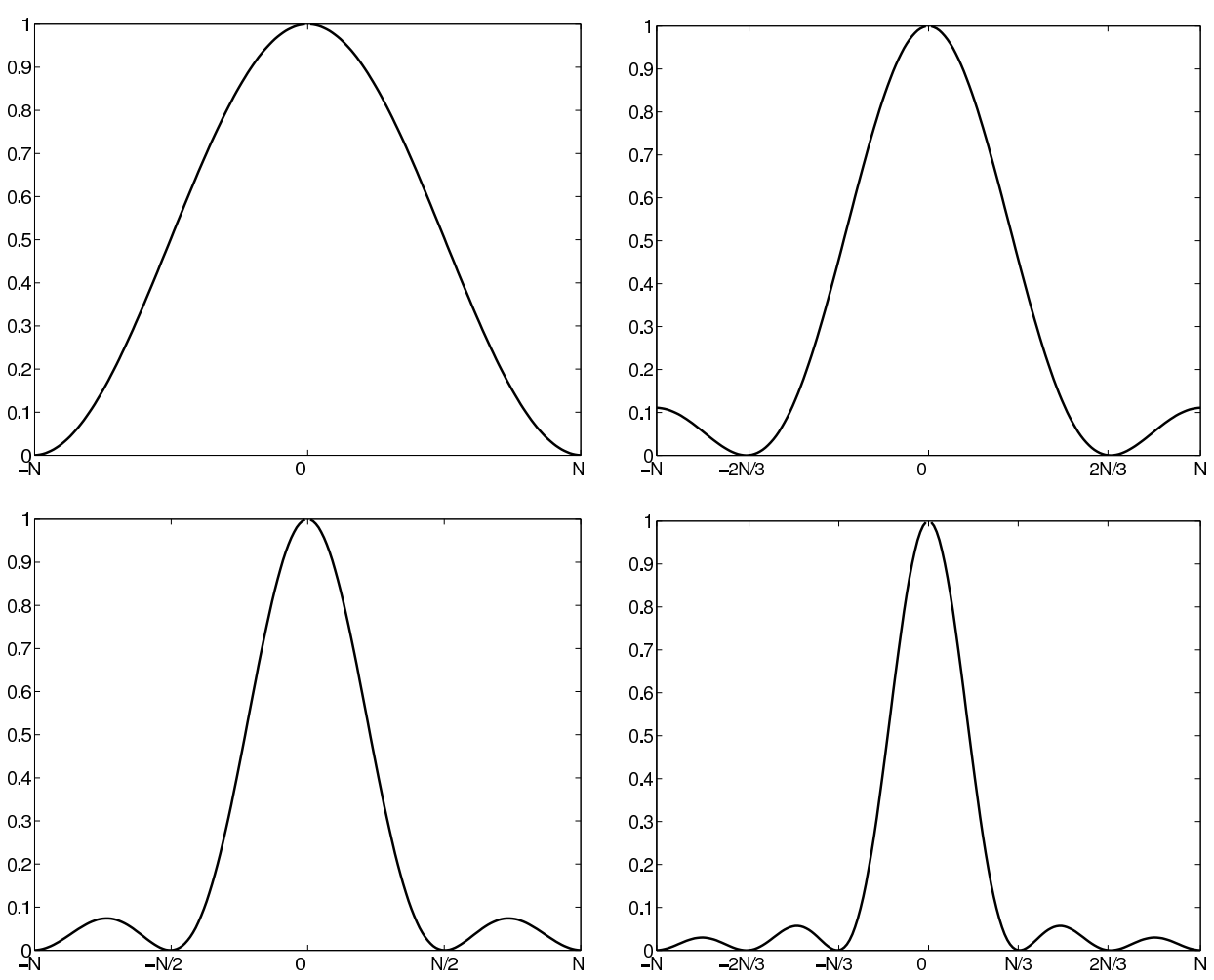

Fig. 7. The multiplicative factors generated by the two-grid algorithm with mesh-sizes ratio $1 / 2$, $1 / 3,1 / 4,1 / 6$ respectively.

Lemma B.1. Let $p \geq 2, N, \tilde{N}$ positive integers such that $2 N=p \tilde{N}$, let $h=2 /(2 N+1)$, and consider the discrete function $v(p k), k \in \Lambda_{\tilde{N}}$. Then the discrete function $u(k)$, $k \in \Lambda_{2 N}$, obtained from the linear interpolation of $v, u=\mathbf{P}_{h}^{1} v$, has the Fourier coefficients satisfying

$$
\widehat{u}(\mathbf{j})=e^{i(p-1)\left(j_{1} h+\cdots+j_{d} h\right) \pi} \prod_{l=1}^{d}\left(p^{-1} \sum_{k=0}^{p-1} e^{i k \pi j_{l} h}\right)^{2} \widehat{v}(\mathbf{j}), \quad \mathbf{j}=\left(j_{1}, \ldots, j_{d}\right) .
$$

In particular, for any $\mathbf{j}$,

$$
|\widehat{u}(\mathbf{j})| \simeq p^{-2 d}|\widehat{v}(\mathbf{j})| \prod_{r=1}^{d}\left|\frac{e^{-i p \pi j_{r} h}-1}{e^{-i \pi j_{r} h}-1}\right|^{2} .
$$

Proof. We will analyze the one-dimensional case. Iterating the same argument in each space direction shows that the same holds in several space dimensions. In this case, we write $u$ explicitly:

$$
u(k p+j)=\frac{(p-j) v(k p)+j v((k+1) p)}{p}, \quad k=0, \ldots, \tilde{N}-1, j=0, \ldots, p-1 .
$$


The $k$-th Fourier coefficient of $u$ is given by

$$
\widehat{u}(j)=h \sum_{k=0}^{2 N} u_{j} e^{-i \pi j k h}, \quad k=-N, \ldots, N
$$

Explicit computation gives

$$
\begin{aligned}
\widehat{u}(j) & =h \sum_{k=0}^{\tilde{N}-1} \sum_{r=0}^{p-1} e^{-i \pi j(k p+r) h} u(k p+r) \\
& =h \sum_{k=0}^{\tilde{N}-1} \sum_{r=0}^{p-1} e^{-i \pi j(k p+r) h} \frac{(p-r) v(k p)+r v((k+1) p)}{p} \\
& =\frac{h}{p} \sum_{k=0}^{\tilde{N}-1} e^{-i \pi j k p h} v(k p)\left(\sum_{r=0}^{p-1} e^{-2 i \pi j r h}(p-r)+\sum_{r=0}^{p-1} e^{i \pi j(p-r) h} r\right) \\
& =\widehat{v}(j) e^{i \pi(p-1) h}\left(p^{-1} \sum_{r=0}^{p-1} e^{-i \pi j r h}\right)^{2} .
\end{aligned}
$$

In particular,

$$
|\widehat{u}(j)| \simeq p^{-2}|\widehat{v}(j)|\left|\frac{e^{-i p j \pi h}-1}{e^{-i \pi j h}-1}\right|^{2} .
$$

Proof of Lemma 3.1. Since for any $\mathbf{j}$ with $\|\mathbf{j}\|_{\infty} \leq N / p$ we have

$$
p^{-2 d} \prod_{r=1}^{d}\left|\frac{e^{-i p \pi j_{r} h}-1}{e^{-i \pi j_{r} h}-1}\right|^{2} \simeq 1
$$

we get

$$
\left\|\Upsilon_{h}^{1 / p} u\right\|_{\mathcal{H}_{h}^{s}}^{2} \simeq \sum_{\|\mathbf{j}\|_{\infty} \leq N / p} \lambda_{\mathbf{j}}^{2 s}|\widehat{v}(\mathbf{j})|^{2}
$$

We split the $\mathcal{H}_{h}^{s}$-norm of $u$ as follows:

$$
\begin{aligned}
\|u\|_{\mathcal{H}_{h}^{s}}^{2}= & \sum_{\|\mathbf{j}\|_{\infty} \leq N} \lambda_{\mathbf{j}}^{2 s}(h) p^{-4 d} \prod_{r=1}^{d}\left|\frac{\exp \left(-i p \pi j_{r} h\right)-1}{\exp \left(-i \pi j_{r} h\right)-1}\right|^{4}|\widehat{v}(\mathbf{j})|^{2} \\
\leq & p^{-4 d} \sum_{\|\mathbf{j}\|_{\infty} \leq N / p} \lambda_{\mathbf{j}}^{2 s}(h)|\widehat{v}(\mathbf{j})|^{2} \\
& +p^{-4 d} \sum_{N / p \leq\|\mathbf{j}\|_{\infty} \leq N} \lambda_{\mathbf{j}}^{2 s}(h) \prod_{r=1}^{d}\left|\frac{\exp \left(-i p \pi j_{r} h\right)-1}{\exp \left(-i \pi j_{r} h\right)-1}\right|^{4}|\widehat{v}(\mathbf{j})|^{2}
\end{aligned}
$$




$$
\begin{aligned}
& \leq c(p, d) \sum_{\|\mathbf{j}\|_{\infty} \leq N / p} \lambda_{\mathbf{j}}^{2 s}(h)|\widehat{v}(\mathbf{j})|^{2} \\
& \quad+c(p, d) h^{-2 s} \sum_{N / p \leq\|\mathbf{j}\|_{\infty} \leq N} \prod_{r=1}^{d}\left|\frac{\exp \left(-i p \pi j_{r} h\right)-1}{\exp \left(-i \pi j_{r} h\right)-1}\right|^{4}|\widehat{v}(\mathbf{j})|^{2} \\
& \leq c(p, d)\left(I_{1}+I_{2}\right) .
\end{aligned}
$$

We prove that for any $\mathbf{j}$ with $N / p \leq\|\mathbf{j}\|_{\infty} \leq N$,

$$
\prod_{r=1}^{d}\left|\frac{\exp \left(-i p \pi j_{r} h\right)-1}{\exp \left(-i \pi j_{r} h\right)-1}\right|^{4} \leq \sum_{r=1}^{d}\left|\exp \left(-i p \pi j_{r} h\right)-1\right|^{2 s}
$$

Let us suppose that $j_{1}=\|\mathbf{j}\|_{\infty} \geq N / p$. Thus $\left|\exp \left(-i \pi j_{1} h\right)-1\right| \geq c_{0}>0$ with $c_{0}$ independent of $h$. Using the inequality

$$
\left|\frac{e^{-i p \xi}-1}{e^{-i \xi}-1}\right| \leq p
$$

for any $\xi \in(-\pi, \pi)$, we obtain

$$
\begin{aligned}
\prod_{r=1}^{d}\left|\frac{\exp \left(-i p \pi j_{r} h\right)-1}{\exp \left(-i \pi j_{r} h\right)-1}\right|^{4} & \leq p^{d-1}\left|\frac{\exp \left(-i p \pi j_{1} h\right)-1}{\exp \left(-i \pi j_{1} h\right)-1}\right|^{4} \\
& \leq c(p, d)\left|\exp \left(-i p \pi j_{1} h\right)-1\right|^{4} \\
& \leq c(p, d, s)\left|\exp \left(-i p \pi j_{1} h\right)-1\right|^{2 s}
\end{aligned}
$$

provided that $s \leq 2$.

Then, using the periodicity of the coefficients $\widehat{v}(\mathbf{j})$ and of $\exp \left(-i p \pi j_{r} h\right)$, we get

$$
\begin{aligned}
I_{2} & \leq c(p, d, s) \quad \sum_{N / p \leq\|\mathbf{j}\|_{\infty} \leq N}|\widehat{v}(\mathbf{j})|^{2} \sum_{r=1}^{d}\left|\frac{\exp \left(-i p \pi j_{r} h\right)-1}{h}\right|^{2 s} \\
& =\left(p^{d}-1\right) c(p, d, s) \sum_{\|\mathbf{j}\|_{\infty} \leq N / p}|\widehat{v}(\mathbf{j})|^{2} \sum_{r=1}^{d}\left|\frac{\exp \left(-i p \pi j_{r} h\right)-1}{h}\right|^{2 s} \\
& \leq c(p, d, s) \sum_{\|\mathbf{j}\|_{\infty} \leq N / p}|\widehat{v}(\mathbf{j})|^{2} \sum_{r=1}^{d}\left|\frac{\exp \left(-i \pi j_{r} h\right)-1}{h}\right|^{2 s} \\
& \leq c(p, d, s) \sum_{\|\mathbf{j}\|_{\infty} \leq N / p} \lambda_{\mathbf{j}}^{2 s}|\widehat{v}(\mathbf{j})|^{2} .
\end{aligned}
$$

The proof is now complete.

Acknowledgments. This work is supported by the Grant MTM2008-03541 of the MICINN (Spain), grant RP-3, no. 4-01/10/2007 of the Romanian MEC and the SIMUMAT project of the CAM (Spain). 


\section{References}

[1] Asch, M., Lebeau, G.: Geometrical aspects of exact boundary controllability for the wave equation-a numerical study. ESAIM Control Optim. Calc. Var. 3, 163-212 (1998) Zbl 1052.93501 MR 1624783

[2] Bardos, C., Lebeau, G., Rauch, J.: Sharp sufficient conditions for the observation, control, and stabilization of waves from the boundary. SIAM J. Control Optim. 30, 1024-1065 (1992) Zbl 0786.93009 MR 1178650

[3] Burq, N.: Contrôlabilité exacte des ondes dans des ouverts peu réguliers. Asymptot. Anal. 14, 157-191 (1997) Zbl 0892.93009 MR 1451210

[4] Burq, N.: Contrôle de l'équation des ondes dans des ouverts comportant des coins. Bull. Soc. Math. France 126, 601-637 (1998) Zbl 0937.35097 MR 1693461

[5] Burq, N., Gérard, P.: Condition nécessaire et suffisante pour la contrôlabilité exacte des ondes. C. R. Acad. Sci. Paris Sér. I Math. 325, 749-752 (1997) Zbl 0906.93008 MR 1483711

[6] Burq, N., Gérard, P., Tzvetkov, N.: Strichartz inequalities and the nonlinear Schrödinger equation on compact manifolds. Amer. J. Math. 126, 569-605 (2004) Zbl 1067.58027 MR 2058384

[7] Castro, C., Micu, S.: Boundary controllability of a linear semi-discrete 1-D wave equation derived from a mixed finite element method. Numer. Math. 102, 413-462 (2006) Zbl 1102.93004 MR 2207268

[8] Castro, C., Micu, S., Münch, A.: Boundary controllability of a semi-discrete 2-D wave equation with mixed finite elements. Preprint.

[9] Glowinski, R.: Ensuring well-posedness by analogy; Stokes problem and boundary control for the wave equation. J. Comput. Phys. 103, 189-221 (1992) Zbl 0763.76042 MR 1196839

[10] Glowinski, R., Kinton, W., Wheeler, M. F.: A mixed finite element formulation for the boundary controllability of the wave equation. Int. J. Numer. Methods Engrg. 27, 623-635 (1989) Zbl 0711.65084 MR 1036928

[11] Glowinski, R., Li, C. H., Lions, J.-L.: A numerical approach to the exact boundary controllability of the wave equation. I. Dirichlet controls: description of the numerical methods. Japan J. Appl. Math. 7, 1-76 (1990) Zbl 0699.65055 MR 1039237

[12] Glowinski, R., Lions, J.-L.: Exact and approximate controllability for distributed parameter systems. In: Acta Numerica, 1995, Cambridge Univ. Press, Cambridge, 159-333 (1995) Zbl 0838.93014 MR 1352473

[13] Ignat, L. I., Zuazua, E.: A two-grid approximation scheme for nonlinear Schrödinger equations: dispersive properties and convergence. C. R. Math. Acad. Sci. Paris 341, 381-386 (2005) Zbl 1079.65090 MR 2169157

[14] Infante, J. A., Zuazua, E.: Boundary observability for the space semi-discretizations of the 1-D wave equation. Math. Model. Numer. Anal. 33, 407-438 (1999) Zbl 0947.65101 MR 1700042

[15] Lebeau, G.: Contrôle de l'équation de Schrödinger. J. Math. Pures Appl. 71, 267-291 (1992) Zbl 0838.35013 MR 1172452

[16] Lebeau, G.: The wave equation with oscillating density: observability at low frequency. ESAIM Control Optim. Calc. Var. 5, 219-258 (2000) Zbl 0953.35083 MR 1750616

[17] León, L., Zuazua, E.: Boundary controllability of the finite-difference space semi-discretizations of the beam equation. ESAIM Control Optim. Calc. Var. 8, 827-862 (2002) Zbl 1063.93025 MR 1932975

[18] Lions, J.-L.: Contrôlabilité exacte, perturbations et stabilisation de systèmes distribués. Tome 1, Recherches en Math. Appl. 8, Masson, Paris (1988) Zbl 0653.93002 MR 0953547 
[19] Lions, J.-L., Magenes, E.: Problèmes aux limites non homogènes et applications. Vols. 1-2, Travaux et Recherches Math. 17, Dunod, Paris (1968) Zbl 0165.10801 MR 0247243

[20] Loreti, P., Mehrenberger, M.: An Ingham type proof for a two-grid observability theorem. ESAIM Control Optim. Calc. Var., to appear

[21] Macia, F.: Propagación y control de vibraciones en medios discretos y continuos. Tesis de doctorado, Univ. Complutense de Madrid (2002)

[22] Micu, S.: Uniform boundary controllability of a semi-discrete 1-D wave equation. Numer. Math. 91, 723-768 (2002) Zbl 1002.65072 MR 1912914

[23] Miller, L.: Controllability cost of conservative systems: resolvent condition and transmutation. J. Funct. Anal. 218, 425-444 (2005) Zbl 1122.93011 MR 2108119

[24] Negreanu, M., Zuazua, E.: Uniform boundary controllability of a discrete 1-D wave equation. Systems Control Lett. 48, 261-279 (2003) Zbl pre05055499 MR 2020643

[25] Negreanu, M., Zuazua, E.: Convergence of a multigrid method for the controllability of a 1-D wave equation. C. R. Math. Acad. Sci. Paris 338, 413-418 (2004) Zbl 1038.65054 MR 2057174

[26] Negreanu, M., Zuazua, E.: Discrete Ingham inequalities and applications. SIAM J. Numer. Anal. 44, 412-448 (2006) Zbl pre05029819 MR 2217389

[27] Quarteroni, A., Valli, A.: Numerical Approximation of Partial Differential Equations. Springer Ser. Comput. Math. 23, Springer (1994) Zbl 0803.65088 MR 1299729

[28] Ramdani, K., Takahashi, T., Tenenbaum, G., Tucsnak, M.: A spectral approach for the exact observability of infinite-dimensional systems with skew-adjoint generator. J. Funct. Anal. 226, 193-229 (2005) Zbl pre02207236 MR 2158180

[29] Russell, D. L., Weiss, G.: A general necessary condition for exact observability. SIAM J. Control Optim. 32, 1-23 (1994) Zbl 0795.93023 MR 1255956

[30] Simon, J.: Compact sets in the space $L^{p}(0, T ; B)$. Ann. Mat. Pura Appl. (4) 146, 65-96 (1987) Zbl 0629.46031 MR 0916688

[31] Zuazua, E.: Boundary observability for the finite-difference space semi-discretizations of the 2-D wave equation in the square. J. Math. Pures Appl. 78, 523-563 (1999) Zbl 0939.93016 MR 1697041

[32] Zuazua, E.: Propagation, observation, control and numerical approximation of waves approximated by finite difference methods. SIAM Rev. 2, 197-243 (2005) Zbl 1077.65095 MR 2179896

[33] Zuazua, E.: Control and numerical approximation of the wave and heat equations. In: Proc. ICM 2006, M. Sanz-Solé et al. (eds.), Vol. III, "Invited Lectures", Eur. Math. Soc., 1389-1417 (2006) Zbl 1108.93023 MR 2275734 